\title{
Savoirs professionnels et prévention des TMS : réflexions conceptuelles et méthodologiques menant à leur identification et à la genèse de leur construction
}

Professional know-how and MSD prevention: conceptual and methodological reflection leading to their identification and the start of their construction Saberes profesionales y prevención de LME : reflexiones conceptuales y metodológicas que conducen a su identificación y a la génesis de su construcción

\section{Sylvie Ouellet et Nicole Vézina}

\section{OpenEdition \\ Journals}

Édition électronique

URL : http://journals.openedition.org/pistes/2251

DOI : 10.4000/pistes. 2251

ISSN : 1481-9384

Éditeur

Les Amis de PISTES

Édition imprimée

Date de publication : 1 novembre 2008

Référence électronique

Sylvie Ouellet et Nicole Vézina, «Savoirs professionnels et prévention des TMS : réflexions conceptuelles et méthodologiques menant à leur identification et à la genèse de leur construction », Perspectives interdisciplinaires sur le travail et la santé [En ligne], 10-2 | 2008, mis en ligne le 21 novembre 2008, consulté le 20 avril 2019. URL : http://journals.openedition.org/pistes/2251 ; DOI : $10.4000 /$ pistes. 2251

Ce document a été généré automatiquement le 20 avril 2019.

Pistes est mis à disposition selon les termes de la licence Creative Commons Attribution - Pas d'Utilisation Commerciale - Pas de Modification 4.0 International. 


\title{
Savoirs professionnels et prévention des TMS : réflexions conceptuelles et méthodologiques menant à leur identification et à la genèse de leur construction
}

\author{
Professional know-how and MSD prevention: conceptual and methodological \\ reflection leading to their identification and the start of their construction \\ Saberes profesionales y prevención de LME : reflexiones conceptuales y \\ metodológicas que conducen a su identificación y a la génesis de su construcción
}

Sylvie Ouellet et Nicole Vézina

\section{Introduction}

Des transformations importantes ont marqué le monde du travail au cours des dernières décennies entraînant l'implantation de nouvelles formes d'organisation du travail, la recherche de polyvalence chez le personnel et la création de nombreuses situations d'apprentissage pour les travailleurs (Rouilleault, H., Rochefort, T. (2005) ; Murray et collab., 2004 ; Laflamme, 2002 ; Everaere, 1999 ; Dubé et Mercure, 1997 ; Sperandio, 1996). La formation de la main-d'œuvre est devenue un enjeu majeur pour les gestionnaires qui accordent de plus en plus d'importance aux savoir-faire développés par leurs employés (Doray et Dubar, 2004). Ces changements peuvent expliquer que, dans les domaines de la santé au travail et de l'ergonomie, il y ait un intérêt grandissant pour cette notion de savoir-faire, particulièrement pour les «savoir-faire de prudence » qui permettent aux travailleurs de se protéger. Cet intérêt s'est reflété dans plusieurs études qui ont visé, d'une part, à décrire les savoir-faire qui permettent aux travailleurs de faire face aux 
contraintes rencontrées et protéger leur santé (Denis et collab., 2007; Chassaing, 2006; Ouellet et collab., 2003 ; Chatigny, 2001a ; Lachance, 2000 ; Authier, 1996 ; Gaudart ; 1996) et, d'autre part, à montrer l'utilité de connaître les savoirs construits afin d'élaborer un contenu de formation qui favorise le développement des savoir-faire chez les novices ou apprentis (Vézina et collab., 1999). L'étude des savoir-faire pose cependant un défi considérable aux chercheurs parce qu'ils sont très difficiles à décrire, entre autres à cause de la difficulté des travailleurs à formaliser leurs façons de faire (Teiger, 1996, 1993 ; Daniellou et Garrigou, 1995; Reber, 1989). Deux facteurs pourraient expliquer cette difficulté soit : 1 - le développement d'automatismes dans l'activité qui rendent plusieurs savoirs de métier inconscients offrant ainsi la disponibilité cérébrale nécessaire pour la planification et l'anticipation des situations critiques (Leplat, 2005); 2- le manque d'occasions pour ces travailleurs de formaliser leurs savoirs (Vézina et collab., 1999). L'étude présentée dans le présent article vise à faire avancer la réflexion sur cette question. Mentionnons que cette étude a été réalisée à l'intérieur d'un projet de doctorat et s'inscrit dans la suite des travaux déjà réalisés par des chercheures du CINBIOSE (Chatigny, 2001b ; Vézina, 1999). L'objectif général du projet est de faire avancer les connaissances sur la relation entre l'organisation de la formation, les conditions d'apprentissage, le contenu de formation et l'acquisition de nouvelles habiletés motrices. Bien qu'au cours de l'étude une intervention ait été réalisée dans le but d'apporter des transformations à l'organisation de la formation et aux conditions d'apprentissage, nous traiterons dans cet article que de la partie portant sur la conception du contenu de formation.

2 L'étude a été réalisée dans une entreprise qui compte au total 350 employés de production dont 25 qui effectuent le dégraissage et le désossage de fesses de porc. Ces travailleurs, tous masculins et droitiers, ont une moyenne d'âge de 41,8 \pm 7,3 ans. Il y a quelques années, une réorganisation du travail a mené à la parcellisation des tâches. L'étude fait suite à une demande de l'entreprise qui voulait obtenir un contenu de formation au désossage de pièces de viande et se faire guider dans la façon d'organiser sa formation dans le but de prévenir les TMS qui représentaient un problème important. Les travailleurs effectuent la transformation de la fesse de porc en séquences sur une chaîne de production mobile. La cadence générale est de 18 secondes, c'est-à-dire qu'à chaque période de 18 secondes il y a une pièce de viande droite ou gauche qui tombe de façon aléatoire sur le convoyeur. Pour les besoins de cet article, seule la tâche de désossage a été considérée. Cette tâche comprend deux séquences, soit une première qui consiste à enlever l'os de la hanche, séquence appelée "pelvis » et une deuxième séquence qui consiste à enlever les deux os de la patte reliés par une jointure, séquence appelée " fémur ». Cette dernière séquence a fait l'objet de notre étude puisqu'elle est la plus longue et qu'elle a été désignée par les travailleurs comme étant la plus difficile à apprendre. Sur la chaîne, nous retrouvons trois postes de travail côte à côte qui sont dédiés à la séquence "fémur ». Chaque désosseur sur ces postes transforme une pièce de viande sur trois, ce qui alloue à chacun d'eux 54 secondes pour effectuer une séquence qui comprend les opérations suivantes : 1- placer la pièce de viande ; 2 - désosser la pièce de viande ; 3- faire la finition du morceau intérieur ; 4- replacer la pièce de viande et 5affiler le couteau. Puisque l'étude porte sur le désossage, nous avons seulement présenté, dans cet article, le temps pour l'opération 2, "désosser la pièce de viande ». Onze travailleurs sont appelés à occuper ce poste puisqu'il y a rotation de postes sur la chaîne. 


\section{Cadre théorique}

3 Mentionnons d'abord que la construction de ce projet de recherche en ergonomie a été faite à partir d'une intégration de plusieurs concepts et modèles provenant de différents domaines : l'ergonomie (Guérin et collab., 2006; Chatigny, 2001 ; Vézina, 2001; Bourgeois et collab., 2006) pour rendre compte de l'activité de travail, la physiologie (Bouisset, 2002) pour aborder la relation contraintes-astreintes liée à la complexité de la tâche et du geste et la neurophysiologie pour comprendre les mécanismes de contrôle du mouvement et de l'apprentissage moteur (Schmidt, R.A., Lee T.D., 2005; Berthoz, 1997; Latash, M.L.; Turvey, M.T., 1996). Nous aborderons dans cet article, à l'aide de la démarche ergonomique, la description des savoir-faire et des savoirs construits par les travailleurs expérimentés. Dans cette démarche, l'activité de travail sera considérée dans sa globalité en tenant compte de l'individu avec ses caractéristiques spécifiques, son statut et ses représentations, en recherchant les différents déterminants que sont l'entreprise avec ses règles de fonctionnement et les conditions de réalisation du travail ainsi qu'en décrivant la tâche comme résultat anticipé et fixé.

4 Tel que mentionné en introduction, l'étude des savoir-faire n'est pas chose simple parce qu'ils sont difficiles à décrire. En ce sens, Jones et Wood (cités par Rasse, 1991) mentionnent que « leurs origines (ils se constituent dans le domaine de l'informel), leur mode de transmission (au moyen d'une pédagogie fondée sur la démonstration et l'observation où la parole et l'écrit se réduisent au minimum indispensable), leur quotidienneté (ils sont acquis à force d'habitude, de gestes et de réflexes mille fois reproduits) font que les employés qualifiés en ont rarement conscience et ne parviennent à en parler que sommairement, de façon allusive et pauvre. " Mais que se cache-t-il derrière cette notion de savoir-faire? Vouloir les décrire ne suppose-t-il pas aussi de pouvoir en cerner la signification? De nombreuses tentatives ont été faites en ce sens sans qu'il y ait consensus sur une définition précise (Le Boterf, G., 2006 ; Minet, 2005 ; Chatigny et Vézina, 2004 ; Garrigou et collab., 2004 ; Chatigny, 2001 ; Danis et Solar, 1998 ; de Montmollin, 1997; de Terssac, 1996; Stroobants, M., 1993; Pelegrin, 1991; Rasse, 1991; Chevallier, D., 1991; Chevallier, 1989). Ainsi, pour certains auteurs, la notion de savoir-faire fait référence à des connaissances (générales, opératoires, routinières), à leurs modes d'utilisation et aux modalités de leur acquisition (de Terssac, 1996) ou à des connaissances procédurales qui se manifestent dans l'activité (de Montmollin, 1997). Pour d'autres, la notion de savoir-faire correspond à une "capacité", soit à des capacités opérationnelles, imbriquées, acquises par la participation au processus de travail (Barcet et collab., 1983, cité par Stroobants, 1993, p.49), soit à la capacité d'effectuer des opérations mentales et à des estimations sur les résultats de ces opérations (Pelegrin, 1991), ou bien à la capacité de réussir une activité particulière par un engagement personnel (Deforge, 1991). Cependant, il n'est pas énoncé clairement dans ces écrits comment un individu peut arriver à avoir cette « capacité » de réussir ou d'effectuer des opérations.

Quant à la notion de « savoir-faire de prudence », elle semble tout aussi difficile à décrire si l'on en juge les quelques définitions présentées dans la littérature. D'abord, mentionnons que sans que ce soit toujours exprimé de façon explicite, les auteurs semblent tous suggérer que les "savoir-faire de prudence " correspondent à des pratiques informelles qui vont dans le sens de la sécurité ou de la protection de la santé. 
Par exemple, pour Cru et Dejours (1983, cité par Garrigou et collab., 2004, p. 507), les savoir-faire de prudence sont un ensemble d'attitudes, de comportements, de façons d'opérer, qui vont dans le sens de la sécurité alors que Chatigny et Vézina (2004) mentionnent que «le développement de savoir-faire de prudence ne consiste pas simplement à appliquer individuellement des règles de prudence, mais surtout à développer, en relation avec le milieu, ses propres stratégies de prudence, les meilleurs compromis permettant de maintenir l'équilibre entre soi-même, les autres et le système de production. » De leur côté, Garrigou et collab. (2004) présentent une définition plus détaillée de la notion de savoir-faire de prudence en présentant des exemples de ce que nous pouvons aussi appeler des stratégies développées par les travailleurs. Ainsi, ils mentionnent que ces savoir-faire

" peuvent porter sur différents aspects, tant au niveau individuel que collectif : la préparation des opérations à risques ; les manières de faire pendant les opérations ; le maintien de l'attention et de la vigilance ; l'anticipation d'aléas ou de variabilités dans les situations de travail; des parades ou des "trucs" pour percevoir, ressentir, gérer le stress ou l'énervement; la vérification et la prise de recul par rapport aux opérations réalisées; la coordination des opérations impliquant différents acteurs; la surveillance des difficultés rencontrées par les autres opérateurs, par exemple les nouveaux embauchés; la transmission au sein du groupe d'histoires d'incidents qui entretiennent la vigilance. »

Mais pouvons-nous penser que toutes les stratégies adoptées par des opérateurs dans un but de protection de la santé sont nécessairement efficaces en ce sens? Si tel n'est pas le cas, pouvons-nous toujours parler de savoir-faire de prudence? Qu'est-ce qui nous permet de parler de savoir-faire de prudence, est-ce l'objectif visé de se protéger ou son efficacité ? Du point de vue de Vidal-Gomel (2002),

« un savoir-faire de prudence est soit fonctionnellement aussi efficace qu'une règle

de sécurité, s'il s'y substitue, soit un complément à la règle de sécurité. »

7 Mais lorsqu'il est question du travail manuel répétitif qui présente des risques élevés de TMS dont les causes sont multifactorielles, est-il possible de parler de savoir-faire de prudence seulement d'un point de vue d'efficacité pour se protéger? Ne serait-il pas plus approprié de considérer la notion de "compromis» qui est le reflet de ce qu'un travailleur considère comme étant le plus efficient possible pour une situation donnée, à un moment donné?

8 En conséquence, malgré l'existence de certaines définitions plus détaillées de la notion de savoir-faire de prudence, elles ne sont peut-être pas encore suffisamment précises pour que l'on puisse savoir exactement comment orienter notre méthodologie lorsqu'il s'agit de vouloir documenter les savoir-faire et les savoir-faire de prudence. Par conséquent, dans cette étude nous voulons aller plus loin dans la réflexion en proposant un cadre théorique et une méthodologie visant à repérer et nommer des savoir-faire de prudence développés par les travailleurs. Cet article vise donc à : 1- proposer une réflexion sur les notions de savoirs, savoir-faire et savoir-faire de prudence; 2- mettre en évidence l'apport de différentes méthodes pour dégager les savoirs des travailleurs expérimentés ; 3- faire ressortir les savoir-faire développés par un groupe de travailleurs expérimentés pour se protéger et 4 - mettre en mots les types de savoirs construits et mobilisés dans la mise en œuvre de ces savoir-faire. Un deuxième article sera présenté dans un numéro ultérieur et visera à vérifier si ces savoirs sont transmis aux apprentis par deux des travailleurs expérimentés qui sont aussi formateurs. 
9 Vouloir faire l'étude des savoir-faire construits par des travailleurs impose donc au préalable de réfléchir à la définition de cette notion qui demeure encore vague malgré le fait qu'elle soit abondamment utilisée dans les écrits en ergonomie et dans d'autres domaines. À la suite de l'analyse des différentes définitions présentées dans la littérature, notre réflexion nous amène à proposer un cadre théorique (figure 1) où un savoir-faire est vu comme la résultante de la mobilisation d'un ensemble de savoirs acquis et développés en amont qui sont continuellement validés et enrichis par la pratique (cercle central). Nous définissons la notion de "savoir-faire» comme étant «la capacité d'un individu à mobiliser dans son activité un ensemble de savoirs lui permettant d'atteindre un objectif ». Avec l'expérience et la pratique, l'individu développe plusieurs savoir-faire qui lui permettent de répondre aux exigences de production et/ou de protéger sa santé. Ce sont ces savoir-faire qui font qu'un travailleur expérimenté pourra se construire une marge de manœuvre. La marge de manœuvre est définie par Durand et collab. (2008) comme étant les possibilités de régulation de l'individu en activité. Elle est la résultante de l'interaction entre les exigences et conditions du travail et les capacités de l'individu. Comme le montre la figure 1, les savoir-faire se manifestent dans l'activité à travers les gestes effectués nous incitant à les présenter par des verbes d'action. Si nous prenons l'exemple de l'affilage du couteau (Vézina et collab., 1999), nous exprimerions un savoirfaire par «affiler son couteau en maintenant le bon angle de la lame sur le fusil». Connaître un "savoir-faire » nécessite donc de connaître le "faire " (Theureau, 1991, p. 43). En conséquence, pour pouvoir repérer les savoir-faire professionnels, il devient incontournable de faire une analyse de l'activité de travail et plus particulièrement l'analyse des gestes mis en œuvre au cours de cette activité. Cette démarche va dans le sens de celle proposée en didactique professionnelle qui est centrée sur le questionnement de l'activité des praticiens (Pastré, 2004). Mentionnons que la signification de la notion de "geste » adoptée ici est celle présentée par Bourgeois et collab. (2006) voulant que le geste soit réfléchi, choisi et adapté en fonction des situations et qu'il résulte d'un compromis entre les objectifs, la tâche, les moyens de travail et les caractéristiques individuelles de celui qui les réalise rendant ainsi compte de l'activité de la personne. Cette notion de gestetient compte non seulement des caractéristiques du mouvement (amplitude, force, vitesse, accélération, etc.), mais aussi des caractéristiques psychologiques (volonté, intention, émotion, etc.) et sociales (poignée de main, « coup de main ", etc.).

10 Dans les études en ergonomie, un savoir-faire permettant de se protéger est habituellement dénommé un "savoir-faire de prudence». Nous adopterons cette appellation pour désigner

«la capacité d'un individu, qui présente une attitude prudente (le «être»), à mobiliser dans son activité un ensemble de savoirs dans le but de protéger sa santé et celle des autres ».

11 Nous considérons qu'il s'agit d'un savoir-faire de prudence lorsque l'intention verbalisée par un travailleur sera de se protéger ou de protéger les autres. À noter que, dans cette étude, la santé est définie comme un

état de complet bien-être physique, mental et social, et non seulement comme une absence de maladie ou d'infirmité (OMS, 1948).

12 Par ailleurs, que peuvent être ces savoirs mobilisés dans les savoir-faire? La figure 1 présente notre point de vue sur la question. Ce point de vue s'est développé en fonction du besoin méthodologique de nommer les éléments que nous avons découverts au 
moment de l'analyse de l'activité de travail et de l'activité de formation des travailleursexperts participant à l'étude.

Figure 1. Modèle de compréhension de la notion de savoir-faire

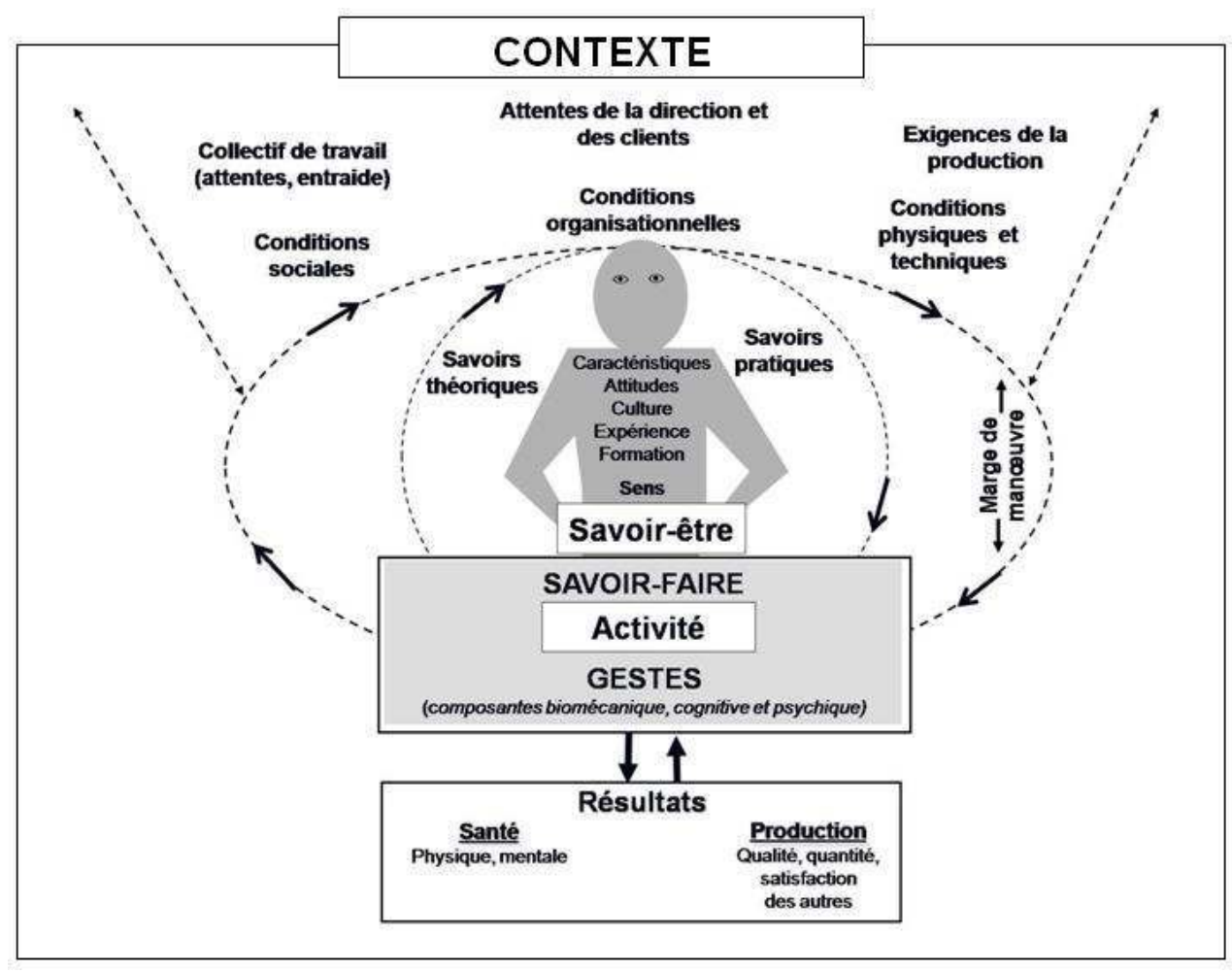

Il est important de préciser que le cadre théorique et la typologie développée sont davantage issus de la pratique sur le terrain que de la fusion de différentes théories issues d'autres domaines, notamment de la didactique professionnelle. Bien que ces théories soient très intéressantes, elles se rapportent beaucoup à la notion de « compétence " pour laquelle il existe différentes définitions. De plus, les interprétations sont variées et il est parfois difficile de bien saisir le lien entre la notion de « compétence » et la notion de "savoir-faire », ce qui mériterait qu'on y accorde plus de place que celle permise dans cet article pour en discuter.

Ainsi, nous proposons une typologie des savoirs mobilisés qui comprend des savoirs théoriques et des savoirs pratiques, la notion de "savoir » étant considérée comme une connaissance acquise par la formation et/ou l'expérience. S'ajoutent à ces deux types de savoirs, les savoir-être qui, comme les savoir-faire, résultent de la relation entre un ensemble de facteurs liés à la personne et pour laquelle nous adoptons la définition donnée par Ketele (cité par Barbier et Galatanu, 2004, p. 59), qui présente les «savoirêtre » comme étant

«les activités par lesquelles la personne manifeste non seulement sa façon

d'appréhender sa propre personne («le concept de soi »), les autres, les situations

et la vie en général, mais aussi sa façon de réagir et d'agir. »

Nous considérons que la personne va construire ses savoir-être dans l'expérience à partir de ses ressources internes (caractéristiques et mémoire des expériences vécues) ainsi que de ce qu'elle sera en mesure de recevoir et de percevoir du collectif. Tout comme les savoir-faire, les savoir-être ne peuvent se transmettre. Cependant, des consignes liées à 
des principes ou des valeurs à privilégier pour réaliser la tâche peuvent être transmissibles, comme par exemple le principe "d'être attentif» ou les trucs pour améliorer la concentration. À partir de ce qui est transmis, l'apprenti tentera de développer cette capacité d'être attentif en essayant d'intégrer ces trucs en fonction de ses caractéristiques, de ses capacités, de son vécu et de sa perception du niveau d'attention requis. L'objectif visé dans cette étude n'est pas de décrire les savoirs nécessaires pour développer les savoir-être des désosseurs puisque nous considérons que cet aspect sort du rôle et du champ de compétences des ergonomes. Néanmoins, nous ne pouvons ignorer cette composante de l'individu dans notre modèle puisque, d'une part, l'approche ergonomique privilégie la considération de la personne réalisant l'activité avec son vécu et ses caractéristiques et, d'autre part, parce que les travailleursformateurs verbalisent des principes liés au " comment être " pour réussir le désossage.

Pour faciliter la compréhension de notre démarche et des résultats qui seront exposés ultérieurement, nous présentons la signification donnée à ces différents types de savoirs. D'abord, mentionnons que les savoirs théoriques sont des connaissances spécialisées relatives à un domaine spécifique qui sont essentiellement acquises par la formation formelle ou informelle. Ce sont donc des savoirs que le formateur va transmettre aux apprentis au cours d'une formation. Ils peuvent se retrouver sous forme écrite dans les manuels et peuvent être enrichis par l'observation et la compréhension d'une pratique qui aura été formalisée. Les "savoirs pratiques", quant à eux, sont des connaissances qui sont développées dans la pratique comme les trucs, les repères, les stratégies, les techniques, les procédures et solutions possibles. Chez les travailleurs expérimentés, ces savoirs découlent d'une comparaison et d'une association entre divers éléments du contexte qui leur permettent de mieux juger et décider des gestes les plus appropriés en fonction des situations. Avec le temps, les «savoirs pratiques » ne sont plus nécessairement conscients et sont difficilement verbalisables. Ils sont évidemment limités chez les novices. Ces savoirs servent à compléter ainsi qu'à relativiser les «savoirs théoriques » et peuvent devenir eux-mêmes des "savoirs théoriques » lorsqu'ils sont formalisés et intégrés à un contenu de formation qui sera transmis à des apprentis. Dans la perspective de faire l'analyse de l'activité des travailleurs expérimentés pour élaborer un contenu de formation, l'objectif est donc d'amener ces derniers à verbaliser leurs savoirs pratiques (donc développés dans leur pratique) pour les rendre transmissibles à la prochaine génération de travailleurs (donc devenir des savoirs théoriques). Bien sûr, l'apprenti aura toujours à développer ses propres savoirs pratiques, car il aura besoin de «mettre en pratique » ses savoirs théoriques et de les adapter à ses caractéristiques personnelles.

Par exemple, lors de l'élaboration de la formation à l'affilage des couteaux (Vézina et collab., 1999), l'identification des savoir-faire par l'analyse de l'activité avait permis de noter que les travailleurs-experts gardaient très droit le poignet de la main qui tient le couteau pendant le passage de la lame sur le fusil (lime fine et allongée). Si on applique ce nouveau cadre théorique à cet exemple, "garder le poignet droit» constitue une connaissance développée dans la pratique, soit un savoir pratique non conscient mais dévoilé par l'analyse des savoir-faire et qui est devenu par la suite un savoir théorique intégré à la formation. Il demeure cependant que l'apprenti doit mettre cette connaissance théorique en pratique (découvrir comment lui-même doit se positionner pour garder le poignet droit) et développer un savoir pratique pour être en mesure de l'utiliser dans l'activité et que ceci fasse partie de ses savoir-faire.

17 Dans la figure 1, nous avons aussi voulu montrer par le cercle central que les savoir-faire se développent par l'enrichissement de tous les types de savoirs mobilisés dans l'activité. 
Par exemple, chaque essai, chaque cycle de travail réalisé, permet au travailleur de juger du résultat, de découvrir de nouveaux repères, de nouvelles solutions permettant ainsi d'enrichir ses savoirs pratiques et, par conséquent, ses savoir-faire. Un savoir-faire ne dépend pas uniquement de la quantité de savoirs possédés, mais surtout de la capacité de l'individu à intégrer et à organiser les savoirs qu'il possède en fonction de soi, du contexte (des conditions techniques, organisationnelles, sociales, etc.) dans lequel l'activité est réalisée. En ce sens, Chevallier et Chiva (1991) cite l'idée de Détienne et Vernant (1974) :

«L'apprentissage du savoir-faire réside aussi dans l'acquisition d'attitudes et d'aptitudes mentales particulières : capacité à prévoir les réactions de la matière, aptitude à conjecturer, caractéristique de la métis analysée par les hellénistes Marcel Détienne et Jean-Pierre Vernant, qui permet de "voir en même temps devant et derrière, c'est-à-dire d'abord avoir l'expérience du passé pour deviner ce qui va se produire, mais aussi rapprocher le futur des événements passés. (p. 5)»

18 Par ailleurs, dans un savoir-faire, tous les sens sont mobilisés (figure 1). Les capacités perceptivo-motrices du travailleur expérimenté lui permettent de pouvoir corriger, ajuster et décider du meilleur geste à effectuer à chacune des étapes de la tâche. En ce sens, Bril et Roux (2002) mentionnent que les caractéristiques de l'expertise ne se situent pas dans la succession des opérations réalisées pour atteindre un but global mais plutôt dans les gestes élémentaires et leur enchaînement (aspect technique) qui sont déterminants d'une expertise qui se caractérise, selon ces auteurs, par les termes de flexibilité et adaptation, anticipation, minimisation de l'énergie, perception et utilisation des informations. Au sujet de la perception, nous adoptons la vision de Berthoz (1997) sur la façon d'aborder l'étude des sens, c'est-à-dire qu'il ne faut pas voir les sens comme des sources d'hypothèses seulement, mais plutôt comme des vérificateurs d'hypothèses que le cerveau pose au monde. Un savoir-faire implique donc de «savoir observer ", «savoir sentir au toucher", "savoir entendre", "savoir humer", "savoir goûter", ce qui implique l'existence de repères. Par exemple, pour savoir sentir au toucher, un travailleur qui cherche à développer un savoir-faire doit connaître ce qu'il y a à sentir, par conséquent connaître des repères. Ainsi, toujours dans la perspective d'élaborer un contenu de formation à partir des savoir-faire des travailleurs expérimentés, il s'avère important de pouvoir dégager les repères que ceux-ci ont développés dans leur pratique (savoirs pratiques). Nous chercherons donc à savoir ce qu'un travailleur vise à vérifier lorsqu'il porte son regard dans une direction ou lorsqu'il touche un objet. Ceci pose un défi considérable puisque qu'il n'est pas toujours facile pour un travailleur expérimenté d'exprimer ses intentions gestuelles, d'une part parce qu'elles sont devenues «mentalement silencieuses » avec le temps et, d'autre part, parce qu'un travailleur n'a pas nécessairement déjà eu l'occasion de verbaliser ses intentions.

Le modèle présenté à la figure 1 a guidé la méthodologie développée pour vérifier nos hypothèses. Mentionnons aussi que plusieurs éléments d'analyse de l'activité proposés dans la méthodologie ont été inspirés des travaux déjà réalisés dans ce secteur par l'équipe de recherche (Vézina et collab., 2002 ; Chatigny, 2001; Ouellet et Vézina, 2001 ; Ouellet, 2000 ; Vézina et collab. 1999 ; Chatigny et Vézina, 1992, 1994 ; Chatigny 1993). Au moyen de cette étude, nous visons contribuer à la prévention des troubles musculosquelettiques par l'élaboration d'un contenu de formation. Considérant que la composante sensorimotrice du savoir-faire fait appel à la mobilisation de différentes modalités sensorielles et au recours à de multiples informations informelles qui permettent aux travailleurs de pouvoir rectifier et choisir le meilleur geste à effectuer, nous formulons l'hypothèse qu'il est nécessaire de faire l'analyse fine des gestes de travail 
pour mettre en mots la genèse de ces régulations. Nous estimons aussi que, dans un objectif de conception de formations, la description des savoir-faire des travailleurs expérimentés doit mener à la mise en mots des savoirs théoriques et pratiques mobilisés pour développer ces savoir-faire puisque ce sont ces savoirs qui pourront ensuite être transmis dans les formations.

\section{Méthodologie: Comprendre le « faire » pour cerner les savoir-faire des travailleurs expérimentés}

\subsection{Le choix des travailleurs-experts}

Ce projet a d'abord débuté par la sélection de six travailleurs expérimentés reconnus par leurs pairs pour leurs savoir-faire. Deux de ces travailleurs expérimentés sont aussi des formateurs au désossage. Le tableau 1 présente les caractéristiques des travailleurs expérimentés qui ont participé à l'étude. Comme nous pouvons le constater, les six travailleurs expérimentés, tous droitiers, sont âgés de 34 à 54 ans pour une moyenne de $44 \pm 7,9$ ans et comptent de 5 à 25 ans d'ancienneté au poste de désossage dans l'entreprise pour une de moyenne $11 \pm 7,7$ ans. Notons que quatre des travailleurs (\#2, \#4, \#5 et \#6) ont occupé pendant plus de 10 ans le même type de postes dans d'autres entreprises du secteur agroalimentaire.

Tableau 1. Caractéristiques des travailleurs experts

\begin{tabular}{|c|c|c|c|c|c|c|}
\hline $\begin{array}{l}\# \\
\text { travailleurs }\end{array}$ & Âge & Taille & $\begin{array}{l}\text { Main } \\
\text { dominante }\end{array}$ & $\begin{array}{l}\text { Ancienneté } \\
\text { usine }\end{array}$ & $\begin{array}{l}\text { Ancienneté } \\
\text { désossage }\end{array}$ & $\begin{array}{l}\text { Autres postes } \\
\text { secteur } \\
\text { viande }\end{array}$ \\
\hline$\# 1^{*}$ & 34 & $\begin{array}{l}1 \mathrm{~m} 73\left(5^{\prime}\right. \\
\left.8^{\prime \prime}\right)\end{array}$ & Droite & 12 ans & 8 ans & Non \\
\hline$\# 2^{*}$ & 42 & $\begin{array}{lll}1 & \mathrm{~m} & 68 \\
\left(5^{\prime} 6^{\prime \prime}\right) & \end{array}$ & Droite & 6,5 ans & 6,5 ans & $\begin{array}{l}\text { Abattage } \\
\text { désossage } \\
20 \text { ans }\end{array}$ \\
\hline$\# 3$ & 36 & $\begin{array}{l}1 \quad \mathrm{~m} \quad 80 \\
\left(5^{\prime} 11^{\prime \prime}\right)\end{array}$ & Droite & 18 ans & 15 ans & Non \\
\hline \# 4 & 50 & $\begin{array}{ll}1 & \mathrm{~m} \\
\left(5^{\prime} 11^{\prime \prime}\right)\end{array}$ & Droite & 10 ans & 5 ans & $\begin{array}{l}\text { Boucher, } \\
22-23 \text { ans }\end{array}$ \\
\hline \# 5 & 47 & $\begin{array}{lll}1 & \mathrm{~m} & 75 \\
\left(5^{\prime} 9^{\prime \prime}\right) & \end{array}$ & Droite & 6,5 ans & 6,5 ans & $\begin{array}{l}\text { Désossage, } 13 \\
\text { ans }\end{array}$ \\
\hline \# 6 & 54 & $\begin{array}{lll}1 & \mathrm{~m} & 73 \\
\left(5^{\prime} 8^{\prime \prime}\right)\end{array}$ & Droite & 25 ans & 25 ans & $\begin{array}{l}\text { Boucher, } 13 \\
\text { ans }\end{array}$ \\
\hline
\end{tabular}

*Travailleurs-formateurs au désossage 


\subsection{Un entretien individuel pour connaître le travail}

21 Par la suite, afin de comprendre le travail réalisé à la séquence "fémur », connaître les difficultés rencontrées ainsi que les malaises ressentis, nous avons tenu un entretien individuel semi-dirigé d'une durée de 60 minutes avec chacun de ces travailleurs. Au moment de l'entretien, aucun des travailleurs n'a mentionné s'être déjà absenté à cause d'une lésion musculo-squelettique associée au poste de désossage. Par contre, un des six travailleurs a mentionné devoir s'absenter de temps en temps à cause de crises aiguës d'arthrite à la cheville droite. Un formulaire de consentement présentant les objectifs et les étapes de l'étude a été signé par tous les travailleurs ayant participé à l'étude. Ceux-ci étaient libres de se retirer de l'étude en tout temps.

\subsection{Des observations au poste de travail}

22 Nous avons ensuite effectué des observations au poste de travail pendant deux jours afin de nous familiariser avec l'organisation du travail, les étapes de la tâche et les différents facteurs pouvant influencer la diversité des modes opératoires. Mentionnons aussi que des verbalisations informelles ont été effectuées durant les observations. Ces verbalisations pouvaient être spontanées de la part des travailleurs ou bien provoquées par l'observateur. Dans le but de faire une description détaillée des modes opératoires et des gestes effectués à chaque étape de la tâche, nous avons réalisé un enregistrement vidéo aux postes de la séquence «fémur " pendant deux autres jours afin d'obtenir deux périodes d'enregistrement de 30 minutes par travailleur.

\subsection{Une analyse fine des gestes professionnels}

23 Avant de faire une analyse systématique des façons de faire, nous avons visionné les séquences vidéo pour caractériser les modes opératoires des travailleurs et définir les variables qui ont servi à l'analyse systématique. Par la suite, l'observation de l'activité de travail et les relevés de données ont été effectués à l'aide du logiciel Actogram (Kerguelen, 2003) destiné à traiter des observations chronologiques à partir de séquences vidéo numérisées qui peuvent être analysées au ralenti. Pour faire l'analyse fine des gestes, nous avons choisi, à partir des enregistrements vidéo, 10 cycles consécutifs sans incident permettant d'obtenir cinq cycles avec la pièce de viande droite et cinq cycles avec la pièce gauche. Dans l'analyse, nous avons considéré : 1- l'organisation temporelle de la tâche (durée: cycle, inter-cycle, étape désossage/étape finition, attente, communication, affilage du couteau, autres) ; 2 - l'ordre des étapes suivies ; 3- la fréquence de certaines actions qui nous apparaissaient déterminantes de l'effort exercé et du temps de cycle (place la pièce, replace la pièce, bouge la pièce) ; 4- la position de la pièce de viande par rapport au travailleur (distance de la pièce et position des parties de la pièce par rapport au travailleur) ; 5- les types de prises sur le couteau en fonction des étapes (prise pleine main, prise index sur le dos de la lame, prise pouce sur le dos de la lame et prise basse poignard) et enfin 6- le pourcentage de temps pour lequel chaque type de prise a été utilisé. Quant aux gestes effectués, notre analyse a porté sur diverses composantes observables telles que : 1 - la direction, l'angle, le nombre et la longueur des coups de couteau; 2- l'amplitude articulaire de certains mouvements de l'épaule, du coude et du poignet, évaluée de façon qualitative et enfin 3- les parties de l'os (zones) où 
se sont situés les coups de couteau. Il est important de mentionner que même si les cycles avec incidents n'ont pas été considérés pour l'analyse gestuelle, les incidents ont été répertoriés et ont fait l'objet de discussion lors des échanges visant à définir les déterminants des méthodes de travail. De plus, nous retrouvons une section dans le contenu de formation pour expliciter l'impact des incidents (ex. : variabilité de la matière première et ses conséquences) et les moyens pour y faire face. Les échanges portant sur ces incidents ont aussi mené, dans certains cas, à des recommandations. Pour les besoins de cet article, seuls les résultats relatifs au temps moyen de désossage, au nombre de coups de couteau, au nombre moyen de déplacements de la pièce de viande et au pourcentage de temps d'utilisation des types de prises sur le couteau seront présentés.

\subsection{L'explicitation des façons de faire par un entretien individuel}

Dans le but de documenter les stratégies utilisées, nous avons tenu une rencontre individuelle d'autoconfrontation d'une durée de 2,5 heures par travailleur pour valider les résultats d'observation obtenus et dégager les déterminants des éléments constituant les méthodes. Pour ce faire, un canevas d'entretien avait été préparé afin de favoriser l'explicitation sur: 1- les étapes de la tâche à effectuer; 2- les différents modes opératoires ; 3- les modalités sensorielles mobilisées ; 4- la qualité du travail atteinte et attendue et 5- la matière première à transformer. Durant cette rencontre, qui était enregistrée, chaque travailleur a d'abord été invité à se regarder sur un enregistrement vidéo et à commenter ce qu'il voyait. Un des six travailleurs n'a pu être rencontré parce qu'il était en congé de maladie prolongé. Cette rencontre a aussi permis de documenter la composante cognitive des gestes effectués (Bourgeois et Hubault, 2005) en documentant les intentions poursuivies par les travailleurs ainsi qu'en indiquant les informations sensorielles recueillies pour anticiper les difficultés et pour corriger, ajuster et décider du meilleur geste à effectuer à chacune des étapes de la tâche. Pour favoriser l'explicitation des informations sensorielles utilisées, nous avions préparé des questions spécifiquement sur cet aspect, comme par exemple : 1- Y a-t-il des éléments que vous regardez lorsque la fesse arrive au poste "fémur »? Si oui, quels sont ces éléments et quelles informations vous fournissent-ils? 2- Utilisez-vous des repères visuels et/ou tactiles qui vous permettent de connaître l'endroit où passer votre couteau pour tracer le morceau « intérieur"? Si oui, quels sont vos repères? (Indiquer les repères sur les photos) 3Lorsque vous dégagez l'os, qu'est-ce qui vous permet de savoir que vous passez votre couteau au bon endroit? 4- Que regardez-vous lorsque vous dégagez l'os? 5- Y a-t-il des repères qui vous permettent de savoir si la lame de votre couteau est enfoncée dans la viande, correctement ou non, pour dégager l'os? Si oui, quels sont ces repères? Le traitement des données obtenues lors de ces rencontres a été effectué à l'aide de l'écoute des enregistrements audio et de l'analyse des notes écrites au cours des entretiens.

\subsection{Une rencontre collective pour se concerter sur les savoirs à transmettre}

Afin de pouvoir répondre à la demande de l'entreprise sur l'élaboration d'un contenu de formation, il nous fallait obtenir un consensus, au sein du groupe de travailleurs, sur les éléments du contenu de formation à la séquence « fémur ». Pour ce faire, nous avons tenu deux journées de rencontre collective enregistrée et filmée pour une durée totale de 14,5 
heures. Cependant, pour les besoins de cet article, seules les données recueillies durant la première journée seront présentées puisqu'elles portent spécifiquement sur les méthodes de travail alors que les discussions de la deuxième journée ont surtout porté sur l'organisation de la formation. Durant cette rencontre, les résultats d'analyse ainsi que les éléments susceptibles d'apparaître dans un manuel de formation ont été présentés de façon illustrée à l'aide de transparents, ce qui a facilité la compréhension des travailleurs et l'expression des savoirs. Les discussions ont porté, entre autres, sur les éléments des différentes méthodes de travail et les repères utilisés pour réaliser la tâche. Pour faciliter l'expression des savoirs, les travailleurs étaient invités à écrire ou à dessiner sur les transparents afin d'expliquer leur façon de faire ou leur point de vue. Il est important de mentionner que l'objectif n'était pas de prescrire des méthodes de travail, mais plutôt d'obtenir un consensus au sein du groupe sur les avantages et les désavantages présentés par les éléments de chacune des méthodes de travail décrites en matière de qualité du produit et de protection de la santé en les faisant ressortir de façon systématique. Pour enrichir les échanges, nous avons organisé, lors de cette rencontre, une période d'essais de 60 minutes dans la salle de production pendant laquelle les travailleurs ont pu démontrer leur façon de faire, essayer les méthodes des autres et donner leur point de vue sur ces méthodes. Il va sans dire que, pour inciter les travailleurs à verbaliser leurs savoirs et montrer leur savoir-faire, il a été important d'établir au début de la rencontre des règles de fonctionnement afin de créer un climat d'échanges les incitant à s'exprimer sans la crainte d'être jugés.

\section{Résultats}

Dans cette section, nous présentons les résultats en trois temps. Nous débutons avec les résultats se rapportant à la tâche réalisée et à la description fine de la gestuelle des travailleurs pour ensuite présenter les savoir-faire de prudence découlant de cette analyse de la gestuelle. Pour terminer, nous décrivons les savoirs qui sont mobilisés dans les savoir-faire répertoriés à l'étape précédente.

\subsection{La tâche de désossage à la séquence " fémur » : la complexité répétée}

Le désossage à la séquence « fémur » consiste à enlever deux os de la fesse de porc reliés par une jointure. Les opérations considérées dans un cycle de travail sont : 1- placer la pièce de viande ; 2 - dégager l'os de la pièce de viande ; 3 - ouvrir et faire la finition du morceau "intérieur » de la pièce ; 4- jeter l'os sur le convoyeur situé en hauteur ; 5replacer la pièce. La figure 2 présente les étapes spécifiques réalisées pour l'opération "dégager l'os de la pièce de viande ». Comme nous pouvons le constater, la première étape consiste à tracer le morceau appelé « intérieur » de la pièce. Le traçage doit se faire obligatoirement en premier sans quoi les autres étapes ne pourront être effectuées. 
Figure 2. Étapes réalisées pour dégager l'os de la pièce de viande à la séquence « fémur »

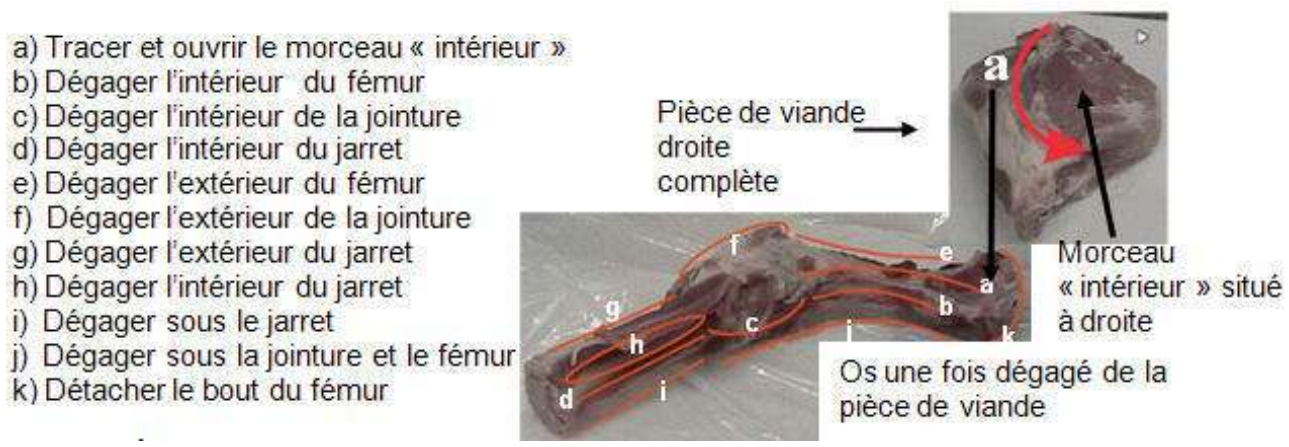

Par contre, le moment de l'ouverture du morceau "intérieur» peut varier d'un travailleur à l'autre. Cette étape consiste à détacher une portion du morceau de la pièce de viande (morceau «intérieur») pour l'étendre sur le convoyeur, d'où l'expression "coucher l'intérieur» souvent utilisée par les travailleurs. Certains travailleurs vont ouvrir le morceau "intérieur » au début de la séquence pour la pièce droite et à la fin pour la pièce gauche à cause de l'ordre d'arrivée de cette partie de la pièce de viande influencé par la direction du convoyeur mobile (figures 3 et 4 ).

Figure 3. Positionnement des parties de la pièce de viande par rapport au travailleur lorsque le convoyeur se dirige de la gauche vers la droite du travailleur

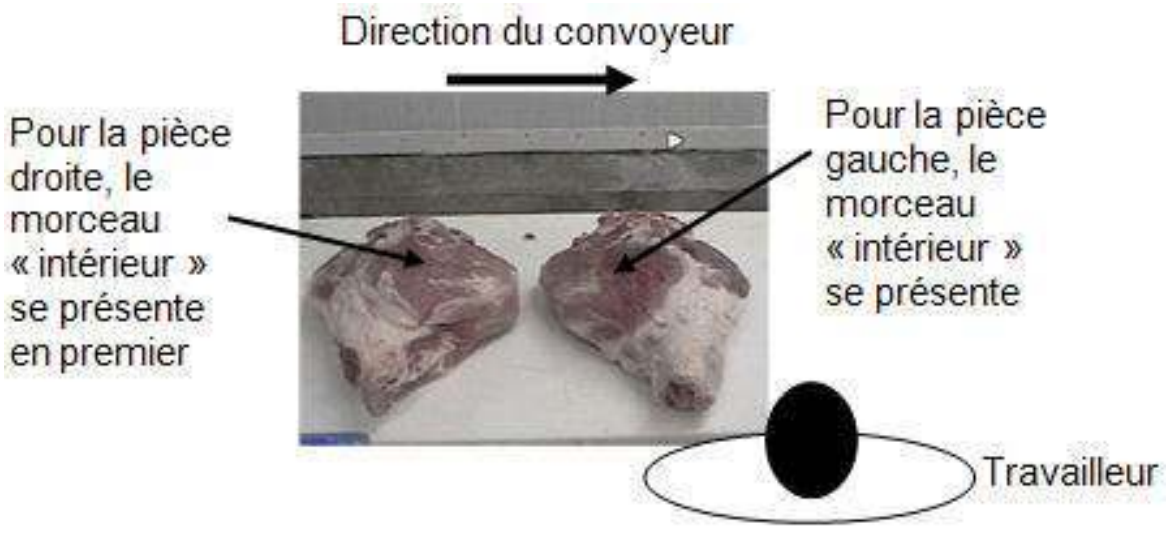

D'autres ouvrent toujours le morceau «intérieur » au début de la séquence. Autre différence à souligner dans l'ordre des étapes, la partie intérieure de l'os (b, c, d) est toujours dégagée avant la partie extérieure $(\mathrm{e}, \mathrm{f}, \mathrm{g})$. Par contre pour l'intérieur du jarret (h), quatre travailleurs sur six vont effectuer cette étape avant l'étape « $\mathrm{e}$ ", un travailleur effectue cette étape après le " $\mathrm{g}$ " pour la pièce droite et avant le « $\mathrm{e}$ " pour la pièce gauche alors que le sixième travailleur effectue toujours cette étape (h) après l'étape " $g$ ». Nous pouvons donc constater que malgré la courte durée du cycle de travail, le fait de faire une analyse fine de la séquence des étapes permet de mettre en évidence une variabilité assez importante dans l'ordre choisi de ces étapes. Nous remarquons aussi que les caractéristiques de la pièce en relation avec les conditions d'exécution du travail (surface mobile et direction du convoyeur) ont une influence notable sur les façons de faire. 


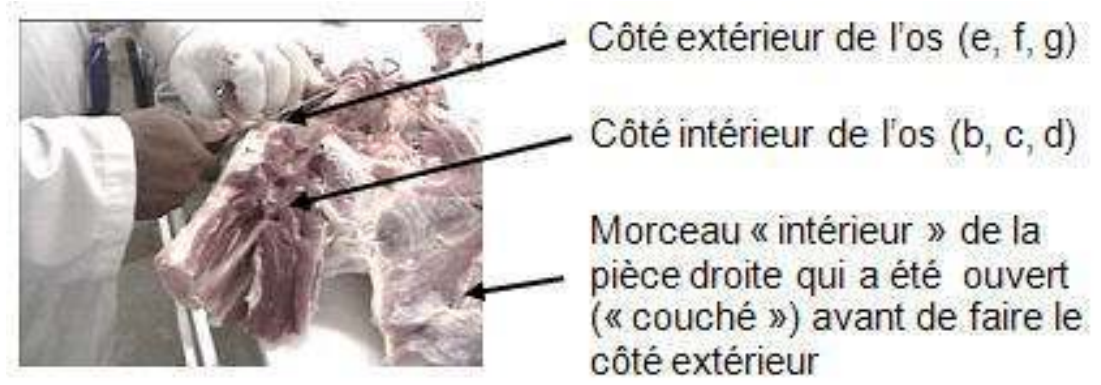

\subsubsection{Penser le travail pour concilier temps, qualité et santé}

Les figures 5 et 6 montrent la relation entre le temps moyen pour « dégager l'os de la pièce de viande ", le nombre moyen de coups de couteau pour dégager l'os et le nombre moyen de déplacements de la pièce de viande. Nous entendons par déplacement de la pièce de viande " un changement de position fait sur le convoyeur sans soulever la pièce ». Nous pouvons d'abord constater que le temps moyen enregistré pour dégager l'os de la pièce de viande a varié de $21,6 \pm 2,7$ à $24,9 \pm 1,8$ secondes pour la pièce droite et de $25,6 \pm 1,6$ à $17,0 \pm$ 1,8 secondes pour la pièce gauche. Nous rappelons ici qu'il ne s'agit pas du temps de cycle mais bien du temps pris pour l'opération de dégager l'os de la pièce. Nous remarquons que les deux travailleurs (\#2,\#3) qui présentent un nombre moyen de coups de couteau plus faible avec 12 à 13 coups (droite et gauche) sont ceux qui présentent un temps moyen pour dégager l'os parmi les plus faibles. En effet, pour ces deux travailleurs, nous avons enregistré un temps moyen de dégagement de $21,6 \pm 2,7$ (\#2) et $23,1 \pm 1,6$ secondes (\#3) pour la pièce droite et de $20 \pm 0,8$ et $17 \pm 1,8$ secondes respectivement pour la pièce gauche. Le travailleur pour qui nous avons enregistré le temps moyen de dégagement le plus long (\#1) avec 24,9 $\pm 1,8$ secondes (droite) et 25,6 $\pm 1,6$ secondes (gauche) est aussi celui qui a donné le plus grand nombre de coups de couteau avec une moyenne de 16,8 (droite) et 17,4 coups (gauche). Notons que les travailleurs \#1 et \#2 sont les deux formateurs de l'entreprise. L'objectif poursuivi par la présentation de ces données n'est pas de savoir s'il existe une différence significative entre les travailleurs ou entre les pièces droites et gauches, mais plutôt de connaître l'amplitude de la variabilité qui peut exister d'un travailleur à l'autre ou d'une pièce à l'autre en se demandant quel impact pourrait avoir cette variabilité sur la sollicitation musculo-squelettique à la fin d'une journée. Par exemple, si nous prenons le cas du nombre de coups de couteau donnés, nous constatons que ceux qui donnent le moins de coups en donnent 12 (travailleurs \#2 et \#3) alors que ceux qui en donnent le plus atteignent près de 17 coups (travailleur \#1). 
Figure 5. Relation entre le temps moyen de dégagement de l'os (sec.), le nombre moyen de coups de couteau et le nombre moyen de déplacements de la pièce durant le dégagement de l'os de la pièce de viande droite

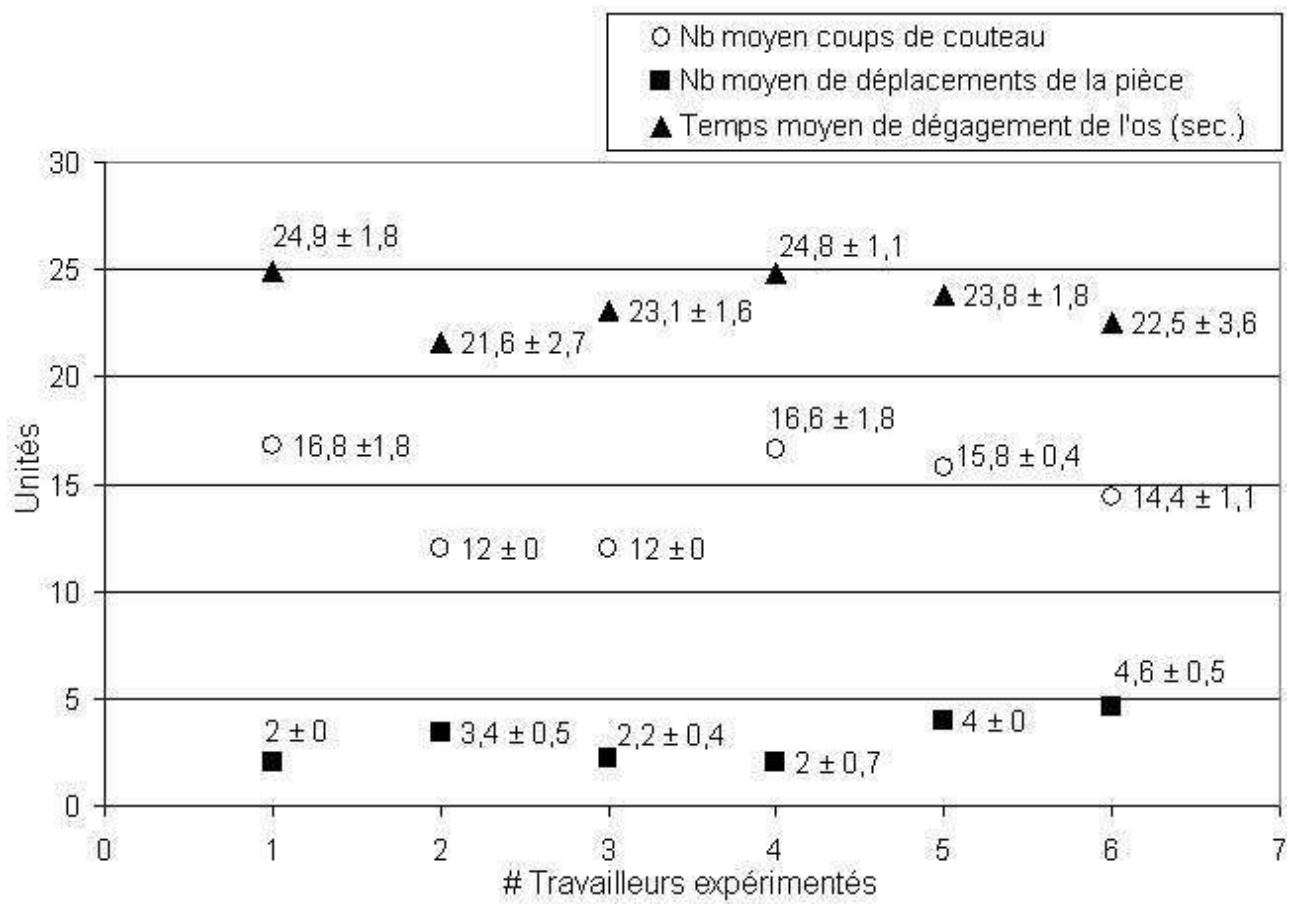

Or, une telle différence pour un travail répétitif avec une cadence prévue de 54 secondes par pièce de viande (cadence de 18 secondes, une pièce sur trois) à cette séquence fait en sorte que le travailleur \#1 donnerait 2475 coups de couteau de plus que les travailleurs \#2 et \#3 s'il demeurait toute la journée à la séquence «fémur » ce qui représente un effort supplémentaire très important. Même un seul coup de couteau de plus représente un supplément de 495 coups de couteau à la fin d'une journée de travail, ce qui est loin d'être négligeable si l'on considère l'effort pouvant être fourni, par exemple lorsque le couteau coupe mal, et les postures adoptées à chaque coup selon les modes opératoires comme, par exemple, les déviations du poignet. Il y a eu consensus au sein du groupe de travailleurs à l'effet que les déterminants possibles du nombre de coups de couteau sont la qualité de coupe du couteau, la méthode de travail et le niveau de précision atteint dans le travail. Au cours des rencontres collectives, les travailleurs ont mentionné qu'il est important de restreindre le nombre de coups de couteau donnés pour diminuer la charge de travail et la perte de temps. Les démonstrations faites par les travailleurs au cours de ces rencontres ont montré qu'il n'est pas nécessaire de donner plus de 12 à 14 coups de couteau pour dégager l'os en respectant les critères de qualité qui sont de retrouver le moins de viande possible sur l'os et aucun déchiquetage sur le morceau de viande. 
Figure 6. Relation entre le temps moyen de dégagement de l'os (sec.), le nombre moyen de coups de couteau et le nombre moyen de déplacements de la pièce durant le dégagement de l'os de la pièce de viande gauche

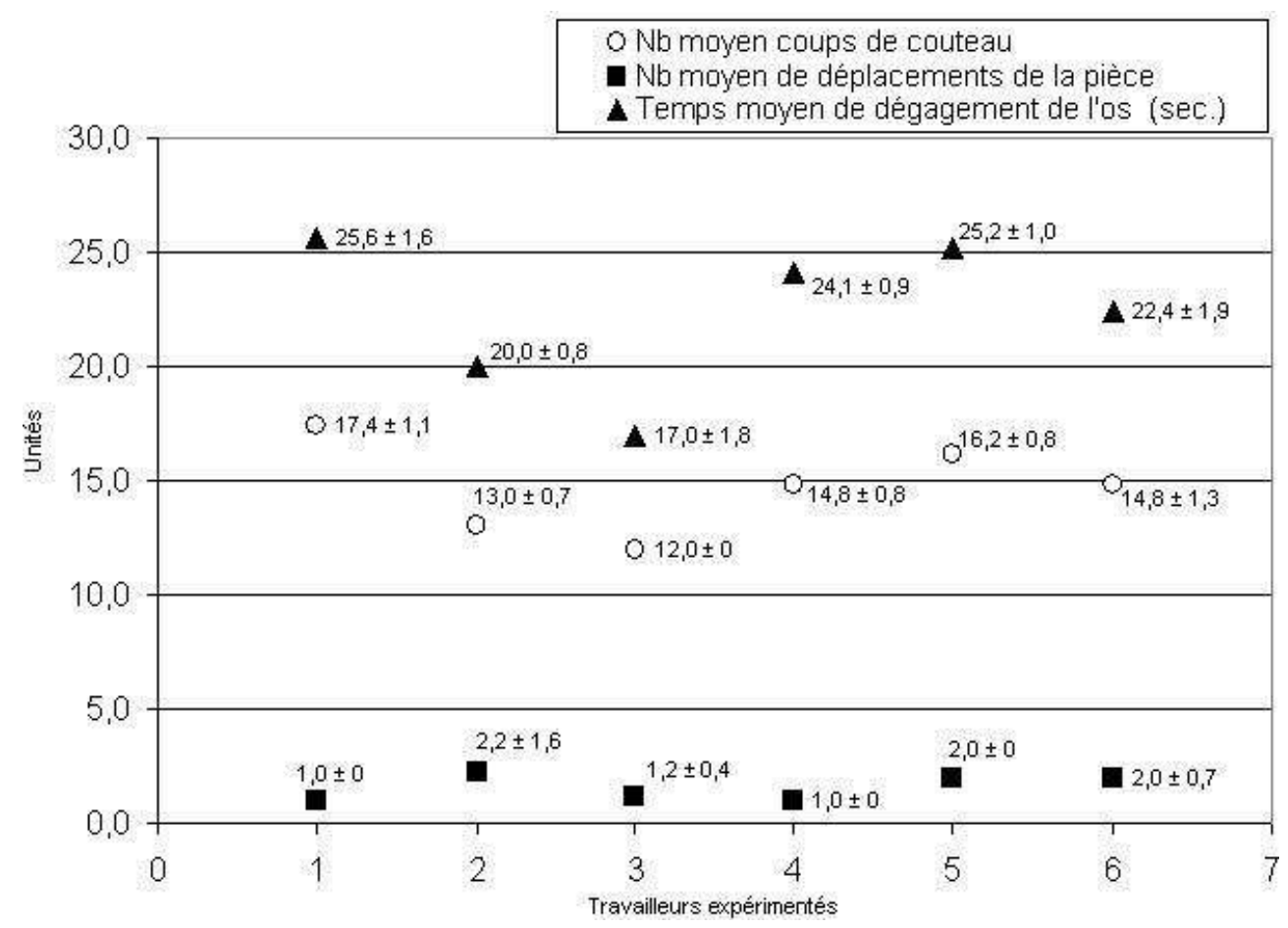

Par ailleurs, il est aussi intéressant de souligner que les travailleurs bougent en moyenne $(1,6 \pm 0,9)$ moins la pièce de viande gauche (figure 6) que la pièce droite $(3,0 \pm 1,1)$ (figure 5). De plus, nous remarquons que les travailleurs \#2 et \#3 qui donnent peu de coups de couteau ne sont pas nécessairement ceux qui bougent le moins la pièce de viande avec une moyenne de $3,4 \pm 0,5$ et $2,2 \pm 0,4$ respectivement pour la pièce droite et $2,2 \pm 1,6$ et 1,2 $\pm 0,4$ pour la pièce gauche. Il est d'ailleurs difficile de voir une relation claire entre le nombre de déplacements et le nombre de coups de couteau. Ce résultat pourrait témoigner des compromis nécessaires à effectuer pour trouver une combinaison d'éléments constituant les méthodes de travail qui permettra à la fois de répondre aux exigences de production tout en protégeant le plus possible la santé. À cet effet, les travailleurs ont mentionné, en rencontre d'autoconfrontation, qu'il fallait faire un compromis entre le fait de ne pas trop bouger la pièce afin de diminuer les efforts et celui de la bouger un peu afin d'être plus à l'aise pour donner les coups de couteau. Nous pouvons donc supposer qu'un principe de prévention donné, comme par exemple "diminuer les mouvements pour diminuer les efforts", ne peut être appliqué à tous les éléments d'une méthode de travail. Les travailleurs doivent faire des compromis entre plusieurs principes pour réussir à obtenir la façon de faire la plus efficace possible en fonction de leur analyse de la situation. De plus, nous pouvons constater que pour la pièce droite (figure 5), parmi les trois travailleurs qui bougent le plus la pièce de viande ( $\# 2$, \#5, \#6), deux présentent les temps moyens de dégagement de l'os parmi les plus faibles (\#2, \#6). Ceci pourrait s'expliquer soit par de petits changements rapides de positions de la pièce qui nécessitent peu de temps ou bien par le fait que certains travailleurs peuvent bouger la pièce en désossant (simultanément), comme l'a mentionné le travailleur \#2 et comme nous l'avons observé. Parmi d'autres différences dans les façons de faire 
observées, nous retrouvons les types de prises utilisées sur le manche du couteau durant le dégagement de l'os. Les types de prises observées sont présentés à la figure 7.

Figure 7. Types de prises sur le manche du couteau utilisées par les travailleurs

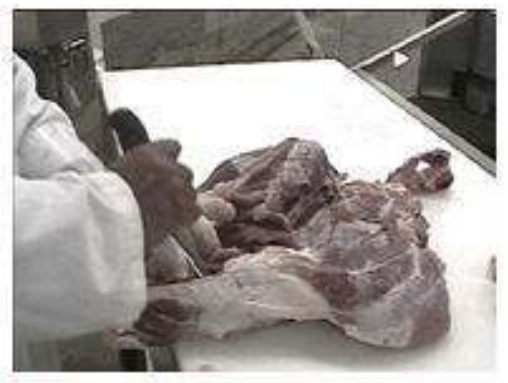

Prise « poignard basse »

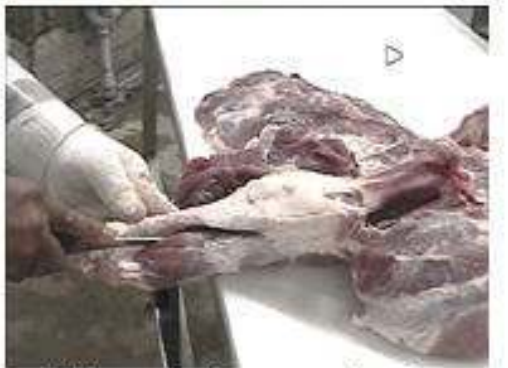

Prise « index sur dos lame»

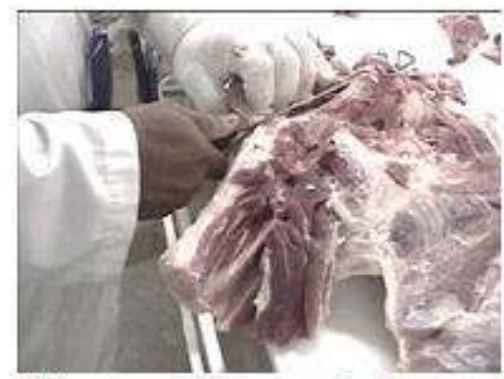

Prise « pouce sur dos lame et manche »

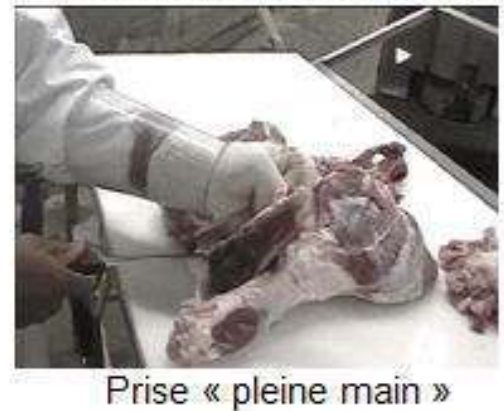

Ainsi, comme le montre le tableau 2, il existe une variabilité importante dans les types de prises adoptées sur le manche du couteau et dans les types de prises privilégiées par rapport à d'autres. Ainsi, alors que la prise pleine main pourrait être considérée comme la prise standard d'un couteau, les données montrent que seul le travailleur \#5 privilégie cette prise par rapport aux autres. Nous constatons, par exemple, que les travailleurs \#1 et \#6 utilisent plus souvent la prise «index sur dos lame » alors que le travailleur \#2 utilise aussi souvent les prises "poignard basse » et «index sur dos lame». Quant au travailleur \#3, il utilise plus souvent la prise « pouce sur dos lame et manche » alors que le travailleur \#4 utilise presque aussi souvent les prises "poignard basse » et "pouce sur dos lame et manche». Les observations montrent en effet que les travailleurs vont changer la prise en fonction des étapes et de la direction du mouvement. Les travailleurs mentionnent utiliser certaines prises comme les prises « index sur dos lame» et " pouce sur dos lame et manche" afin d'obtenir plus de précision et d'être plus à l'aise. Le travailleur \#2 a même mentionné que, pour lui, l'utilisation de cette prise lui fournit la preuve que son couteau coupe bien puisqu'il n'a aucune douleur au doigt. Il ajoute que si son couteau ne coupait pas, il aurait sûrement mal à l'index. De son côté, le travailleur \#5 a mentionné ne pas utiliser ce type de prise parce que le formateur qui l'avait formé, il y a une vingtaine d'années, l'avait empêché de l'utiliser en disant que ce type de prise entraînait des douleurs au poignet. 
Tableau 2. Pourcentage de temps pendant lequel un type de prise a été utilisé, par rapport au temps total enregistré pour tous les types de prises. (Fesses droite et gauche)

\begin{tabular}{|l|l|l|l|l|l|l|l|l|}
\hline \multicolumn{2}{|l|}{$\begin{array}{l}\text { Ordre } \\
\text { ancienneté } \\
\# \text { travailleurs }\end{array}$} & $\begin{array}{l}\text { Temps } \\
\text { total } \\
(\mathrm{sec})\end{array}$ & $\begin{array}{l}\text { Nombre } \\
\text { changements } \\
\text { et (\% temps) }\end{array}$ & $\begin{array}{l}\text { Poignard } \\
\text { basse } \\
\text { temps) }\end{array}$ & $\begin{array}{l}\text { Pleine } \\
\text { main (\% } \\
\text { temps) }\end{array}$ & $\begin{array}{l}\text { Index } \\
\text { dos } \\
\text { lame (\% } \\
\text { temps }\end{array}$ & $\begin{array}{l}\text { Pouce dos } \\
\text { lame/ } \\
\text { manche } \\
\text { (\% temps) }\end{array}$ & $\begin{array}{l}\text { Inconnu } \\
\text { temps }\end{array}$ \\
\hline 1 & $\# 1$ & 328 & $90(12,5)$ & 23,8 & 13,1 & $\mathbf{3 2 , 9}$ & 10,4 & 7,3 \\
\hline 2 & $\# 3$ & 252 & $56(8,3)$ & 10,3 & 11,9 & 18,6 & $\mathbf{5 0 , 0}$ & 0,8 \\
\hline 3 & $\# 5$ & 289 & $72(10,4)$ & 11,8 & $\mathbf{4 5 , 0}$ & 0 & 29,4 & 3,5 \\
\hline 4 & $\# 2$ & 250 & $53(9,2)$ & $\mathbf{3 2 , 0}$ & 2,0 & $\mathbf{3 2 , 0}$ & 22,8 & 2,0 \\
\hline 5 & $\# 4$ & 304 & $75(11,2)$ & $\mathbf{2 5 , 0}$ & 11,2 & 15,1 & $\mathbf{2 7 , 6}$ & 9,9 \\
\hline 6 & $\# 6$ & 279 & $64(10,4)$ & 17,2 & 11,1 & $\mathbf{3 7 , 3}$ & 22,6 & 1,4 \\
\hline
\end{tabular}

Tous s'entendent pour dire qu'il est important de changer de prise en fonction des étapes pour éviter d'adopter des postures contraignantes. Le temps de changement d'une prise a été enregistré au moment où le travailleur relâchait la prise existante jusqu'à l'adoption claire d'une nouvelle prise. Dans le tableau, nous remarquons qu'il y a un certain pourcentage de temps pour lequel les types de prises n'ont pu être identifiés. Ce pourcentage de temps correspond aux moments où il était difficile de voir sur l'enregistrement vidéo si un travailleur utilisait la prise «pleine main» ou «pouce sur dos lame et manche ». Par ailleurs, lorsque nous comparons les données sur les types de prises et les données présentées dans les figures 5 et 6 , nous constatons que les deux travailleurs qui ont changé de prise le moins souvent (\#2 avec 53 changements et \#3 avec 56) sont deux travailleurs qui présentent le temps de dégagement de l'os parmi le plus court et qui donnent le moins de coups de couteau alors que celui qui change de prise le plus souvent (\#1 avec 90 changements) est celui qui présente le temps de dégagement de l'os le plus long (figures 5 et 6), qui donne le plus de coups de couteau et qui bouge le moins la pièce de viande.

Dans cette étude, il nous a été impossible de recueillir des données biomécaniques en simultané avec l'enregistrement vidéo à cause de contraintes liées à l'espace restreint entre les travailleurs (travail à la chaîne) et à la non-disponibilité d'équipements adaptés pour être utilisés dans ce genre de milieux. Ce type de données aurait permis d'approfondir certains aspects avec les travailleurs, ce qui aurait peut-être facilité l'obtention d'un consensus. En revanche, nous pouvons faire l'hypothèse que le fait de changer de prise sur le manche demande du temps, ne serait-ce qu'une fraction de seconde, ce qui pourrait être l'une des raisons expliquant la durée de cycle plus longue pour le travailleur \#1. Nous pourrions aussi nous demander si le fait de ne pas déplacer beaucoup la pièce de viande pourrait entraîner des changements plus fréquents du type de prise. 


\subsubsection{Les sens mobilisés dans le travail : des savoirs du corps insoupçonnés} d'autoconfrontation et collective, nous avons questionné les travailleurs sur les informations sensorielles utilisées avant et pendant le désossage. Le tableau 3 présente les résultats obtenus concernant les informations recherchées en lien avec les sens mobilisés. Lors des verbalisations informelles effectuées au poste de travail durant la collecte de données, les travailleurs avaient souligné qu'une des difficultés du désossage était l'exigence d'être précis dans les gestes effectués surtout à certains endroits dans la pièce de viande qui ne sont pas toujours visibles. Nous avons donc voulu savoir comment les travailleurs faisaient pour rendre leurs gestes plus efficaces. Cinq des objectifs des désosseurs présentés dans le tableau 3 portent sur la question de précision alors que le sixième objectif porte sur les informations utilisées par les travailleurs pour se préparer à chaque cycle (anticiper), «pour préparer le désossage ». Les données montrent d'abord qu'avant chaque cycle (arrivée de la pièce), les travailleurs vérifient visuellement si la pièce est bien placée, si elle est bien nettoyée en amont (enlever le gras) et si c'est une pièce droite ou gauche afin d'anticiper ce qu'ils devront faire avant de commencer à désosser et choisir la méthode qu'ils utiliseront. Un des cinq travailleurs a mentionné qu'il n'avait pas besoin de regarder pour savoir s'il s'agissait d'une pièce droite ou gauche car il lui suffit de toucher la pièce.

Quant aux cinq autres objectifs, nous pouvons constater que les travailleurs utilisent non seulement la vision et la sensation tactile, mais que certains ont aussi mentionné avoir une représentation mentale de la forme et de la dimension de l'os ce qui leur permet d'anticiper les obstacles et de placer leur couteau adéquatement. Nous remarquons aussi que la sensation d'être accoté sur l'os apparaît fréquemment comme étant un moyen pour savoir si le couteau passe à la bonne place avec le bon angle ainsi que pour anticiper les obstacles. Un des travailleurs (\#6, 25 ans d'ancienneté) affirme pouvoir désosser sans regarder et connaître immédiatement le résultat de son travail par la sensation tactile. Un autre (\#2) a mentionné qu'il est important de ne pas trop serrer le couteau pour avoir la sensation tactile de l'os. Mentionnons que ces deux travailleurs sont parmi ceux qui ont le plus d'expérience dans le secteur de la viande.

Les données présentées au tableau 3 mettent en évidence l'importance de la connaissance et de la reconnaissance visuelle ou tactile des caractéristiques de la matière première. Cette constatation pose la question des difficultés d'apprentissage que peuvent rencontrer des apprentis dans ce type de travail qui présente ces exigences perceptivomotrices. Comment favoriser le développement de cette capacité sensorimotrice qu'ont les travailleurs expérimentés sans devoir passer à travers des années de pratique laborieuses et souffrantes? De plus, considérant qu'un couteau qui coupe mal et que le stress du débutant peut entrainer une prise serrée sur le manche, ceci nous laisse croire qu'il serait important d'agir sur certains déterminants avant qu'un travailleur puisse avoir une prise « légère » sur le manche.

Tableau 3. Informations recherchées par les travailleurs au cours de la réalisation de la tâche et ponts avec les connaissances (nombre de travailleurs ayant mentionné cet élément)

\begin{tabular}{|l|l|l|}
\hline Objectifs visés & $\begin{array}{l}\text { Informations recherchées } \\
\text { VÉRIFIER... }\end{array}$ & $\begin{array}{l}\text { Ponts avec les } \\
\text { connaissances }\end{array}$ \\
\hline
\end{tabular}




\begin{tabular}{|c|c|c|c|}
\hline & Visuelles & Tactiles & Représentation mentale \\
\hline $\begin{array}{l}\text { Préparer le désossage } \\
\text { (avant de commencer) }\end{array}$ & $\begin{array}{l}\text { Si la pièce est bien } \\
\text { nettoyée (étape en } \\
\text { amont) (2) } \\
\text { Si la pièce est bien } \\
\text { placée avant de } \\
\text { commencer (4) } \\
\text { S'il y a un abcès (1) } \\
\text { Si c'est une pièce } \\
\text { droite ou gauche } \\
\text { (3) }\end{array}$ & $\begin{array}{l}\text { Forme de la pièce } \\
\text { (savoir si c'est une } \\
\text { pièce droite ou } \\
\text { gauche) (1) }\end{array}$ & \\
\hline $\begin{array}{l}\text { Savoir où passer le } \\
\text { couteau pour tracer le } \\
\text { morceau « intérieur" }\end{array}$ & $\begin{array}{l}\text { Où est la «boule du } \\
\text { fémur " pour } \\
\text { débuter à côté ; } \\
\text { ligne de gras (1) } \\
\text { Où est la «boule du } \\
\text { fémur " pour } \\
\text { débuter en bas } \\
\text { Où est la ligne de } \\
\text { gras pour la suivre } \\
(4)\end{array}$ & $\begin{array}{l}\text { Accotement sur l'os } \\
(1)\end{array}$ & $\begin{array}{l}\text { Forme de l'os sous la } \\
\text { viande (1) }\end{array}$ \\
\hline $\begin{array}{l}\text { Savoir si passe le couteau } \\
\text { au bon endroit }\end{array}$ & Où est l'os (3) & $\begin{array}{lll}\text { Accotement } & \text { sur l'os } \\
(4) & & \\
\text { Texture } & \text { de } & \text { la } \\
\text { viande } & \text { et } & \text { du } \\
\text { cartilage } & \text { qui } & \text { est } \\
\text { différente (1) } & \\
\text { Déformations } & \text { de } \\
\text { l'os (2) } & & \end{array}$ & \\
\hline $\begin{array}{l}\text { Connaître la bonne } \\
\text { profondeur de la lame }\end{array}$ & 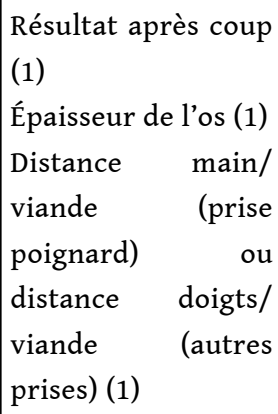 & & $\begin{array}{l}\text { Diamètre et épaisseur de } \\
\text { l'os (2) }\end{array}$ \\
\hline Anticiper les obstacles & & \begin{tabular}{|lrr} 
Sentir & & le \\
changement & & de \\
forme de l'os & qui \\
s'élargit & & avant \\
d'arriver & à & la \\
jointure (1) & &
\end{tabular} & $\begin{array}{l}\text { Distance parcourue par } \\
\text { le couteau par rapport à } \\
\text { lareprésentation } \\
\text { mentale de la longueur } \\
\text { non visible de l'os (1) }\end{array}$ \\
\hline
\end{tabular}




\begin{tabular}{|l|l|l|l|}
\hline $\begin{array}{l}\text { Avoir le bon angle du } \\
\text { couteau }\end{array}$ & Forme de l'os (1) & $\begin{array}{l}\text { Accotement sur l'os } \\
(2)\end{array}$ & Forme de l'os (2) \\
\hline
\end{tabular}

\subsection{L'analyse fine des gestes pour la description des savoir-faire et des savoirs}

\subsubsection{Se construire des savoir-faire pour se protéger} expérimentés ont permis de décrire des savoir-faire et de mettre en mots des savoirs mobilisés pour la mise en œuvre de ces savoir-faire. Dans ces savoir-faire, nous retrouvons ceux qui ont davantage été désignés comme des savoir-faire de "production", c'est-à-dire nécessaires pour répondre aux exigences de qualité, et d'autres qui sont construits par les travailleurs pour se protéger (savoir-faire de prudence) sans exclure la considération des exigences de production. Comme mentionné en introduction, il est coutume dans les études ergonomiques visant la prévention des troubles musculo-squelettiques de vouloir comprendre comment les travailleurs expérimentés arrivent à se protéger en recherchant les savoir-faire de prudence développés par ces derniers. Cependant, cet accent mis sur les savoir-faire de prudence ne risque-t-il pas de négliger la contribution possible de certains savoir-faire désignés "production " pour préserver la santé des travailleurs comme nous laissent croire les résultats obtenus au cours de cette étude ? En effet, alors que certains savoir-faire avaient d'abord été associés à l'atteinte d'un niveau de qualité visé par les travailleurs, ils ont par la suite été mis en relation avec le fait qu'ils permettent de diminuer les efforts et la répétitivité des mouvements en diminuant le nombre de coups de couteau nécessaires. Mentionnons en particulier les savoir-faire " passer son couteau au bon endroit » et "utiliser la partie appropriée de la lame du couteau » qui ont fait consensus auprès des travailleurs expérimentés. Passer le couteau au bon endroit signifie de bien appuyer la lame du couteau sur l'os et passer la lame entre les parties (voies naturelles) de la pièce de viande. Lorsque les travailleurs ont été amenés à verbaliser sur leur méthode de travail lors des rencontres d'autoconfrontation et collective, ils ont expliqué que ce savoir-faire permet, d'une part, de diminuer l'effort puisqu'il y a un mince espace entre chaque partie de la pièce de viande constitué d'une membrane facile à couper et, d'autre part, de diminuer le nombre de coups de couteau puisque ça évite de déchiqueter le gras et les membranes qui doivent être enlevés sur le morceau "intérieur » durant la séquence. Quant au savoirfaire "utiliser la partie appropriée de la lame du couteau», il permet, selon les travailleurs expérimentés, de diminuer l'effort en évitant l'accrochage sur les os, ce qui peut abîmer la lame et augmenter le nombre de coups de couteau. Ce savoir-faire pourrait aussi faciliter le travail des travailleurs au poste en aval (finition) qui doivent nettoyer les morceaux de viande. L'importance de développer ces deux savoir-faire n'est donc pas à négliger si un travailleur veut se protéger.

Par ailleurs, comment nommer les savoir-faire de prudence des travailleurs expérimentés dans ce contexte de travail ? Est-il possible de nommer des savoir-faire de prudence sans allusion à la production puisque ces savoir-faire sont exprimés dans une action de production? Notre réflexion à partir des résultats obtenus et des échanges réalisés avec les travailleurs nous amène à formuler les savoir-faire en débutant toujours avec 
« dégager l'os en... » pour souligner le fait que ce savoir-faire ne prend sa signification que lorsqu'il est exprimé dans l'activité. Étant donné que les résultats obtenus dans cette phase serviront à faire l'analyse de la transmission des savoirs à des apprentis dans une deuxième phase de l'étude, nous avons choisi de présenter les résultats concernant les deux travailleurs-formateurs de l'entreprise. Le tableau 4 présente la liste des savoir-faire de prudence identifiés en fonction de ces travailleurs-formateurs ainsi que les deux savoir-faire davantage associés à la production qui ont été mentionnés précédemment.

Tableau 4. Liste des principaux savoir-faire de prudence et savoir-faire de production pour chacun des deux travailleurs-formateurs

\begin{tabular}{|c|c|c|}
\hline Savoir-faire de prudence & $\begin{array}{l}\text { Travailleur- } \\
\text { formateur } 1\end{array}$ & $\begin{array}{l}\text { Travailleur- } \\
\text { formateur } 2\end{array}$ \\
\hline $\begin{array}{l}\text { Dégager l'os en choisissant le moment d'ouvrir le } \\
\text { morceau «intérieur » en fonction des situations pour } \\
\text { éviter de toucher le collègue en aval }\end{array}$ & $\mathrm{X}$ & $\mathrm{X}$ \\
\hline $\begin{array}{l}\text { Dégager l'os en choisissant les types de prises sur le } \\
\text { couteau en fonction des étapes pour diminuer l'effort et } \\
\text { les postures contraignantes }\end{array}$ & $\mathrm{X}$ & $\mathrm{X}$ \\
\hline $\begin{array}{l}\text { Dégager l'os en dosant le nombre de mouvements du } \\
\text { corps et de la pièce de viande pour être à l'aise et } \\
\text { diminuer l'effort }\end{array}$ & $\mathrm{X}$ & $\mathrm{X}$ \\
\hline $\begin{array}{l}\text { Dégager l'os en donnant le minimum nécessaire de coups } \\
\text { de couteau pour diminuer l'effort }\end{array}$ & & $\mathrm{X}$ \\
\hline $\begin{array}{l}\text { Dégager l'os en positionnant la main non dominante de } \\
\text { façon à éviter les blessures }\end{array}$ & & $\mathrm{X}$ \\
\hline $\begin{array}{l}\text { Dégager l'os en positionnant la pièce de viande ou en se } \\
\text { positionnant de façon à diminuer les postures } \\
\text { contraignantes }\end{array}$ & $\mathrm{X}$ & $\mathrm{X}$ \\
\hline $\begin{array}{l}\text { Dégager l'os en assurant une bonne qualité de coupe du } \\
\text { couteau pour diminuer l'effort }\end{array}$ & $\mathrm{X}$ & $\mathrm{X}$ \\
\hline $\begin{array}{l}\text { Dégager l'os en gérant le temps alloué pour se donner une } \\
\text { marge de manœuvre }\end{array}$ & $\mathrm{X}$ & $\mathrm{X}$ \\
\hline $\begin{array}{l}\text { Dégager l'os en anticipant les difficultés pour se donner } \\
\text { une marge de manœuvre }\end{array}$ & & $\mathrm{X}$ \\
\hline \multicolumn{3}{|l|}{ Savoir-faire de production } \\
\hline $\begin{array}{l}\text { Dégager l'os en passant son couteau au bon endroit pour } \\
\text { assurer une bonne qualité }\end{array}$ & $\mathrm{X}$ & $\mathrm{X}$ \\
\hline
\end{tabular}


Dégager l'os en utilisant la bonne partie de la lame du couteau en fonction des étapes pour assurer une bonne $\mathrm{X}$ qualité

41 Ainsi, nous pouvons remarquer que dans le but de se protéger, les travailleurs-formateurs vont choisir le moment de faire l'étape de l'ouverture de la partie « intérieur » de la pièce de viande, choisir les types de prises sur le couteau en fonction des étapes, doser le nombre de coups de couteau et le nombre de mouvements du corps et de la pièce de viande, se positionner ou positionner la pièce de viande pour éviter les blessures et les postures contraignantes, assurer une bonne qualité de coupe du couteau, gérer le temps et anticiper les difficultés pour se donner de la marge de manœuvre. Soulignons aussi que les deux travailleurs-formateurs présentent les mêmes savoir-faire, sauf celui de " dégager l'os en positionnant la main non dominante de façon à éviter les blessures » et celui de "dégager l'os en donnant le minimum nécessaire de coups de couteau pour diminuer l'effort » qui n'ont pas été observés et exprimés par le travailleur-formateur \#1. Dans la section suivante, nous verrons si ces deux travailleurs-formateurs mobilisent les mêmes savoirs pour mettre en œuvre ces savoir-faire.

\subsubsection{Mobiliser des savoirs pour savoir faire}

L'analyse fine des gestes ainsi que les verbalisations réalisées au cours des rencontres d'auto-confrontation et collective ont aussi permis de définir les types de savoirs mobilisés par les travailleurs-formateurs. Les méthodes utilisées sont l'observation, la rencontre individuelle d'autoconfrontation et la rencontre collective. Soulignons qu'afin de mettre en évidence l'apport que peuvent avoir les rencontres d'autoconfrontation et collective, nous avons dissocié la composante observable du geste comme, par exemple, "ouvrir et nettoyer l'« intérieur » pendant ou après le désossage », de la composante cognitive ou intentionnelle associée: «pour éviter le collègue en aval ». Alors que les observations nous ont permis de pouvoir décrire les actions et les postures, les rencontres individuelle et collective ont permis de mettre en mots les objectifs poursuivis, les repères utilisés, les informations recherchées pour la réalisation de la tâche et d'obtenir un certain raffinement dans l'analyse des actions, difficile à atteindre par l'observation. Ces rencontres ont aussi permis de comprendre comment les travailleurs réussissent à faire face aux contraintes de la production et quelle est la logique derrière certains compromis. Par exemple, pour le savoir« ouvrir et nettoyer l'«intérieur » pendant ou après le désossage", les observations ont permis de constater que le travailleurformateur 1 ouvre le morceau "intérieur " au début de la séquence pour la pièce de viande droite puisque cette partie arrive en premier. Par contre, il ouvre cette partie à la fin de la séquence pour la pièce gauche parce que cette partie "intérieur » arrive en dernier. Le travailleur-formateur 1 mentionne que s'il n'ouvrait pas le morceau « intérieur » de la pièce droite au début (arrive en premier), il risquerait de se retrouver trop loin dans l'espace de son collègue en aval car la pièce se déplace en s'éloignant de lui.

Quant au travailleur-formateur 2, nous avons observé qu'il ouvre le morceau « intérieur " au début de la séquence peu importe que ce soit une pièce droite ou une pièce gauche. Puisque le morceau «intérieur» de la pièce gauche se situe à sa gauche et que le convoyeur se déplace de gauche à droite, le travailleur 2 affirme y voir un certain désavantage à ouvrir le morceau « intérieur » de la pièce gauche au début de la séquence. 
En effet, pendant qu'un travailleur dégage le côté gauche de la pièce et ouvre le morceau « intérieur » qui se situe à sa gauche avant de faire le côté droit, ce dernier s'est éloigné de lui lorsque vient le temps de dégager ce côté. Par contre, si ce travailleur procède de cette façon c'est qu'il y voit l'avantage de sauver du temps puisque, de toute façon, il doit dégager l'intérieur de l'os à gauche qui se trouve aussi du même côté que le morceau « intérieur ». Donc, pour le travailleur-formateur 2, le fait de faire tout ce qu'il y a à faire sur un même côté de la pièce avant de passer ensuite à l'autre côté permet de sauver des secondes. Dans ce métier, où l'on retrouve des cycles de travail très courts, sauver ne serait-ce qu'une seconde revêt une très grande importance pour les travailleurs. Chaque seconde sauvée peut mener à une micro-pause et à une petite marge de manœuvre. Rappelons que le travailleur-formateur 2 est l'un des deux travailleurs ayant le temps moyen de désossage le plus court.

Plusieurs savoirs ont pu être définis comme par exemple : 1- caractéristiques des pièces de viande et de l'os ; 2- position de la pièce de viande près de lui pour éviter les bras tendus ; 3position de la pièce de viande de biais ou position de biais du travailleur pour éviter d'être "pogné et croche »; 4- ordre à suivre dans certaines étapes pour faciliter le travail ; 5 - parties utiles de la lame à utiliser ; 6 - utilisation de longs coups de couteau et 7 - utilisation de la prise pleine main sur le manche, considérée comme la prise standard par les fournisseurs. Mentionnons que les savoirs liés aux caractéristiques des pièces de viande et de l'os ont été qualifiés d'essentiels pour réussir le désossage. Le commentaire du travailleurformateur 1 lors de la rencontre d'autoconfrontation témoigne de cette importance :

«Mais y faut que tu saches comment y est faite ton os. Ben là j'me casse pu la tête avec ça là mais... j'me souviens moi, tant et aussi longtemps que, comme la p'tite, le dessous du jarret, l'os qui est en-dessous complètement qui est incliné un peu,... pis là monsieur $\mathrm{X}$ y m'a enlevé, $\mathrm{y}$ me dit r'garde, y est pas droite,... Mais tant et aussi longtemps qu'il ne me l'a pas montré... »

De plus, nous aimerions porter à l'attention un fait qui illustre bien l'apport qu'a pu avoir la période d'essais pratiques dans la salle de production lors de la rencontre collective. En effet, lors de la rencontre d'autoconfrontation et collective en salle, les travailleurs répétaient fréquemment qu'une des choses importantes pour réussir le désossage était d'utiliser la partie appropriée de la lame du couteau, soit la «pointe » de la lame. Mais en écoutant les travailleurs, deux questions ont émergé du dialogue soit celle de savoir ce que veulent dire les travailleurs en parlant de la « pointe » de la lame et l'autre à savoir si la pointe était véritablement toujours la partie utilisée. Ce dernier aspect était difficilement observable à partir des séquences vidéo. Lors des essais dans la salle de production, nous avons pu mettre en évidence que les travailleurs avaient une représentation différente de ce qu'était pour eux la "pointe » de la lame et que, pour certaines étapes, une partie plus importante de la lame était utilisée. Pour certains, la "pointe» n'était qu'une petite partie de l'extrémité de la lame alors que pour d'autres, cette partie représentait environ le tiers de la longueur de la lame. Pourtant, dans leurs discours, tous disaient que le secret du désossage, c'était de travailler avec la « pointe » de la lame. Les essais pratiques ont donc permis de mettre en lumière la représentation de chacun des travailleurs sur la notion de "pointe» du couteau, de faire prendre conscience à ceux-ci que finalement, dans l'action, ce n'est pas toujours cette partie de la lame qui est utilisée même si c'est effectivement la principale partie concernée et, enfin, de comprendre la logique qui se trouve derrière le choix de la longueur de lame à utiliser en fonction des étapes. 
Par ailleurs, dans une perspective de formation, nous nous sommes questionnées sur la relation pouvant exister, d'une part, entre les savoir-faire et, d'autre part, entre les différents savoirs. Existerait-il des préalables au développement de certains savoir-faire et savoirs? Ce questionnement nous a conduites à la construction d'une chaîne hiérarchique des savoirs et savoir-faire en prenant comme exemple le cas du savoir-faire de prudence « dégager l'os en donnant le minimum nécessaire de coups de couteau » (figure 8). Ainsi, comme nous pouvons le remarquer à la figure 8 , pour posséder ce savoir-faire, les travailleurs expérimentés ont dû développer des savoir-faire préalables davantage associés à la production comme "utiliser la partie appropriée de la lame», "passer au bon endroit » et un savoir-faire davantage associé à la réduction des efforts donc de prudence qui est de "maintenir la coupe de son couteau ». Ces savoir-faire impliquent eux-mêmes la mobilisation de nombreux savoirs à acquérir, à intégrer et/ou à développer comme, par exemple, les caractéristiques de la matière première, les étapes à suivre, les trucs et les repères ainsi qu'un savoir lié au "comment être » pour faciliter le désossage comme le principe "être attentif à l'exécution». Cette chaîne de savoirs et savoir-faire préalables suppose donc que, pour réussir à être prudent, un travailleur a dû d'abord acquérir plusieurs savoirs pour développer d'autres savoir-faire qui permettront de donner moins de coups de couteau. Cette hiérarchie dans les savoirs et savoir-faire peut avoir une incidence importante pour la formation de nouveaux travailleurs.

Figure 8. Chaîne des savoirs et savoir-faire préalables au savoir-faire de prudence " dégager l'os en donnant le minimum nécessaire de coups de couteau »

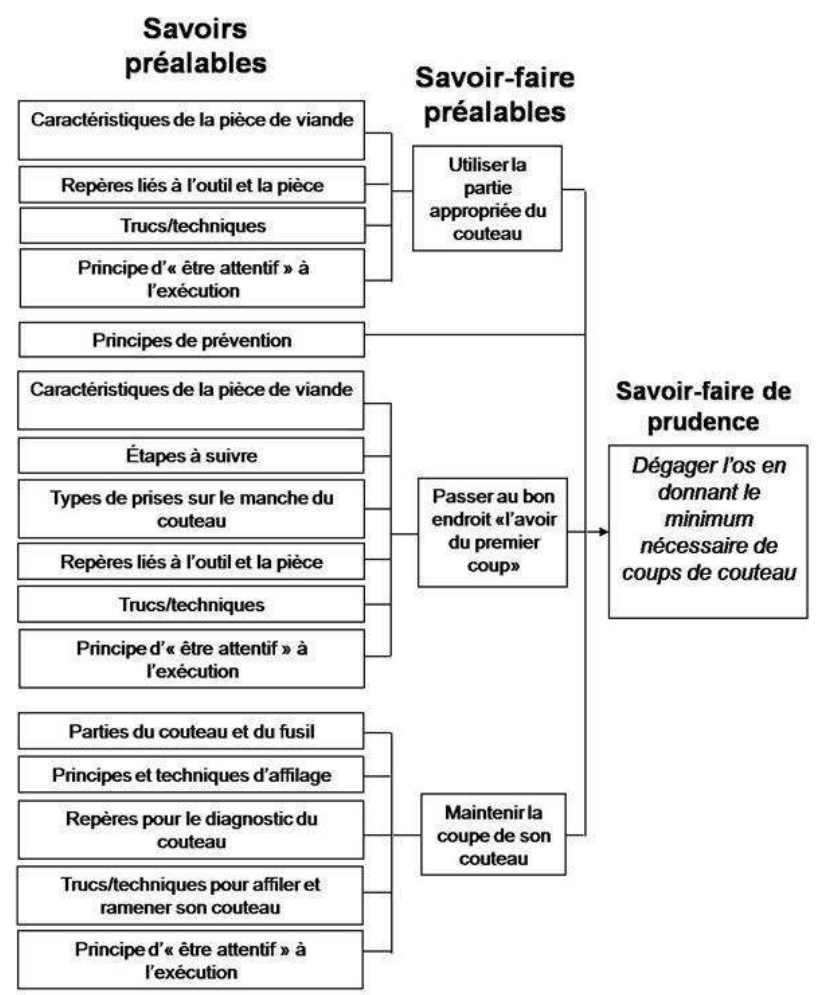

47 Un autre fait intéressant tiré des résultats est qu'un savoir-faire de prudence tel que "dégager l'os en donnant le minimum nécessaire de coups de couteau " peut non seulement avoir une incidence sur la santé mais aussi sur la production. En effet, lorsque nous avons demandé aux travailleurs de nommer les avantages de donner moins de coups de couteau, ceux-ci ont mentionné que ça permet non seulement de diminuer les efforts, 
mais aussi de respecter la cadence et d'avoir un beau produit fini. Il semble donc exister une zone grise entre "savoir-faire de production" et "savoir-faire de prudence". Peuvent-ils exister strictement pour la production ou pour la prudence? Selon les résultats obtenus dans notre étude, il n'y a pas d'évidence en ce sens.

Voulant pousser plus loin l'analyse fine des données, nous avons documenté la relation qui pouvait exister entre certains savoirs. Pour ce faire, nous avons construit une chaîne hiérarchique (figure 9) entre les savoirs qui sont mobilisés pour le savoir-faire « passer au bon endroit » apparaissant précédemment dans la figure 8.

Figure 9. Hiérarchie des savoirs préalables pour le savoir-faire « passer au bon endroit » avec le couteau

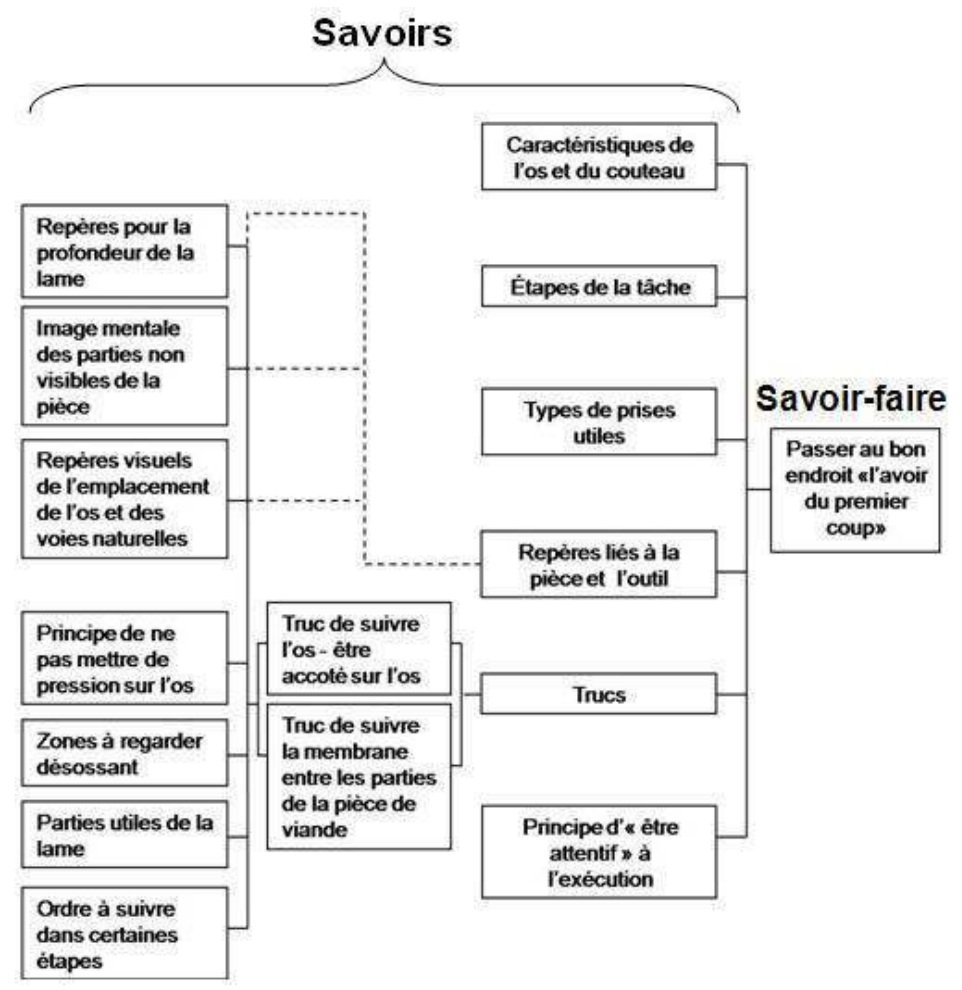

Comme le montre la figure 9, deux trucs peuvent être transmis pour arriver à passer au bon endroit soit: «suivre l'os - être accoté sur l'os » et «suivre la membrane entre les parties de la pièce de viande ». La question à laquelle nous avons voulu répondre est: «Est-ce qu'il y a des savoirs qui doivent être transmis si nous voulons que ces trucs permettent la mise en œuvre du savoir-faire? Nous remarquons dans la figure qu'effectivement il a fallu développer d'autres savoirs pour arriver à développer ces trucs de métier, comme par exemple: 1- parties utiles de la lame; 2- ordre à suivre dans certaines étapes ; 3 - principe de ne pas mettre de pression sur le manche du couteau, etc. Ainsi, ces deux dernières figures mettent en évidence l'existence d'une certaine hiérarchie dans le développement des savoir-faire et des savoirs qui doit être prise en compte lorsque nous voulons favoriser le développement de savoir-faire. 


\section{Discussion}

\subsection{Formaliser les savoirs et les savoir-faire des travailleurs expérimentés : nécessité ou option?}

Le travail manuel répétitif est souvent perçu comme un travail peu complexe et pour lequel la composante biomécanique attire davantage l'attention. Cette perception mène souvent à la croyance qu'une tâche répétitive peut s'apprendre rapidement et qu'il suffit d'observer les autres travailleurs pour pouvoir réaliser cette tâche. Pourtant, l'analyse fine des gestes et les verbalisations réalisées avec les travailleurs ont permis de mettre en évidence que ceux-ci ont construit, avec le temps et la pratique, des savoirs qui leur permettent de mettre en œuvre des savoir-faire visant à résoudre les problèmes posés au quotidien, à se construire une marge de manœuvre ainsi qu'à se protéger, protéger les autres et répondre aux exigences de production comme l'ont montré Teiger et Laville (1989). La variabilité enregistrée dans les modes opératoires montre comment chacun de ces travailleurs expérimentés s'est approprié les façons de dégager l'os en fonction de ses caractéristiques, de ses capacités, de ses valeurs et de sa compréhension des situations rencontrées.

51 Pourquoi vouloir formaliser les savoirs et les savoir-faire des travailleurs expérimentés? Dans plusieurs entreprises, particulièrement dans le secteur agroalimentaire, la formation est de plus en plus vue comme un moyen de prévenir les troubles musculosquelettiques. Dans ce contexte, les travailleurs expérimentés sont souvent amenés à agir comme formateurs alors qu'on leur demande de transmettre des connaissances qu'ils ont acquises, développées et incorporées dans leur pratique. Ces connaissances sont reliées à la réalisation de l'activité elle-même (gestes, perception sensorimotrice, planification), mais aussi aux caractéristiques de la matière première qui doit être "travaillée ", aux outils à utiliser et aux diverses conditions à prendre en compte. Tel que mentionné précédemment, les connaissances liées aux modes opératoires ont été développées en fonction des caractéristiques des travailleurs. Puisque les connaissances détenues par un travailleur expérimenté ne sont pas nécessairement adaptées aux caractéristiques d'un apprenti, il devient important de pouvoir formaliser les connaissances de plusieurs travailleurs expérimentés afin d'offrir aux apprentis la possibilité de choisir celles qui seront les plus adaptées à leurs caractéristiques.

D’un autre côté, des écrits (Teiger,1996; Daniellou et Garrigou,1995; Reber, 1989) font état de la difficulté des travailleurs à verbaliser leurs savoirs lorsque nous les questionnons sur leurs façons de faire, notamment sur les savoirs pratiques qui sont incorporés et parmi lesquels nous retrouvons les savoirs mobilisés dans les savoir-faire de prudence. Cette difficulté de verbalisation ne risque-t-elle pas de se retrouver aussi lorsqu'un travailleur sera amené à former des apprentis? En conséquence, l'objectif de prévenir les TMS par la formation pourra-t-il vraiment être atteint? Ceci nous laisse croire que l'atteinte de cet objectif de prévention doit passer par la formalisation des savoirs et des savoir-faire des travailleurs expérimentés pour permettre : 1- de rendre les savoirs, particulièrement les savoirs pratiques, transmissibles afin de favoriser le développement des savoir-faire de prudence ; 2 - de définir les connaissances qui sont pertinentes pour la réalisation d'une tâche et, par conséquent, de définir de nouvelles formes de documentation technique et de donner les moyens d'améliorer la 
compréhension du travail à réaliser ; 3- de décrire les exigences de la tâche dans toutes ses composantes (biomécanique, cognitive et psychologique) et de documenter les connaissances que les apprentis doivent acquérir et s'approprier pour arriver à réaliser la tâche tout en se protégeant, et enfin 4- de prévoir les difficultés d'apprentissage d'une tâche et d'organiser la formation en conséquence.

\subsection{Les savoir-faire professionnels : y a-t-il des préalables à leur construction?}

53 Au-delà de l'importance de la composante physique, l'activité de désossage, pour être menée à bien, demande aux travailleurs de saisir un grand nombre d'informations et de faire preuve de maîtrise sur leur environnement et sur eux-mêmes. De plus, contrairement à ce qui peut parfois être perçu, les travailleurs ne sont pas passifs face aux risques liés à leur travail. Ils développent des savoir-faire de prudence et des stratégies pour les gérer (Cru, 1995). La construction des savoir-faire nécessite la mobilisation d'un ensemble de savoirs qui ont été acquis par la formation formelle ou informelle ou construits dans la pratique. Par exemple, la figure 10 présente une synthèse de l'ensemble des savoirs mobilisés pour être capable de "passer le couteau au bon endroit ", savoir-faire davantage associé à la qualité de production mais qui permet aux travailleurs d'exercer moins de force pour couper la pièce et donner moins de coups de couteau pour atteindre un niveau de qualité donné.

Pour développer ce savoir-faire, un travailleur doit acquérir et mobiliser des savoirs théoriques mais aussi développer des savoirs pratiques qui viendront valider et enrichir avec le temps ces savoirs théoriques. Mentionnons toutefois qu'en absence de formation, il est possible que ces savoirs théoriques doivent être acquis «sur le tas ", donc dans la pratique. Parmi les savoirs théoriques, nous retrouvons principalement la connaissance des caractéristiques de la matière première et des équipements disponibles (ex.: noms et localisation des parties de la pièce, types de couteaux disponibles, etc.), la connaissance des critères de qualité et des principes de prévention et la connaissance des procédures. Quant aux savoirs pratiques, nous retrouvons entre autres les répertoires de solutions possibles, de repères et de trucs de métier (ex: repères pour la profondeur de la lame), le répertoire de techniques (ex. : relation types de prises sur le couteau/étapes/types de déplacements de la pièce de viande) de même que la mémoire d'expériences vécues (ex. : " telle façon de faire m'a donné mal au poignet »). Ces savoirs peuvent s'acquérir lors de formation formelle ou informelle lorsqu'ils sont formalisés, mais dans le cas de nos travailleurs-experts, ils ont été presque essentiellement construits dans la pratique. De plus, des savoir-être peuvent contribuer à la mise en œuvre des savoir-faire qui permettront aux travailleurs d'atteindre l'objectif visé. Parmi les savoirêtre verbalisés par les travailleurs et présentés à la figure 10, nous retrouvons la concentration, l'attention, la réceptivité à apprendre et à développer de nouvelles façons de faire, ainsi que la prudence. Le cercle en pointillé reliant les savoirs et savoir-être vise à montrer l'influence mutuelle que peuvent avoir les savoirs ainsi que les savoir-être des travailleurs. 
Figure 10. Modèle de mobilisation d'un ensemble de savoirs mobilisés dans le savoir-faire efficient " passer le couteau au bon endroit en positionnant la pièce de viande de façon à diminuer les contraintes posturales"

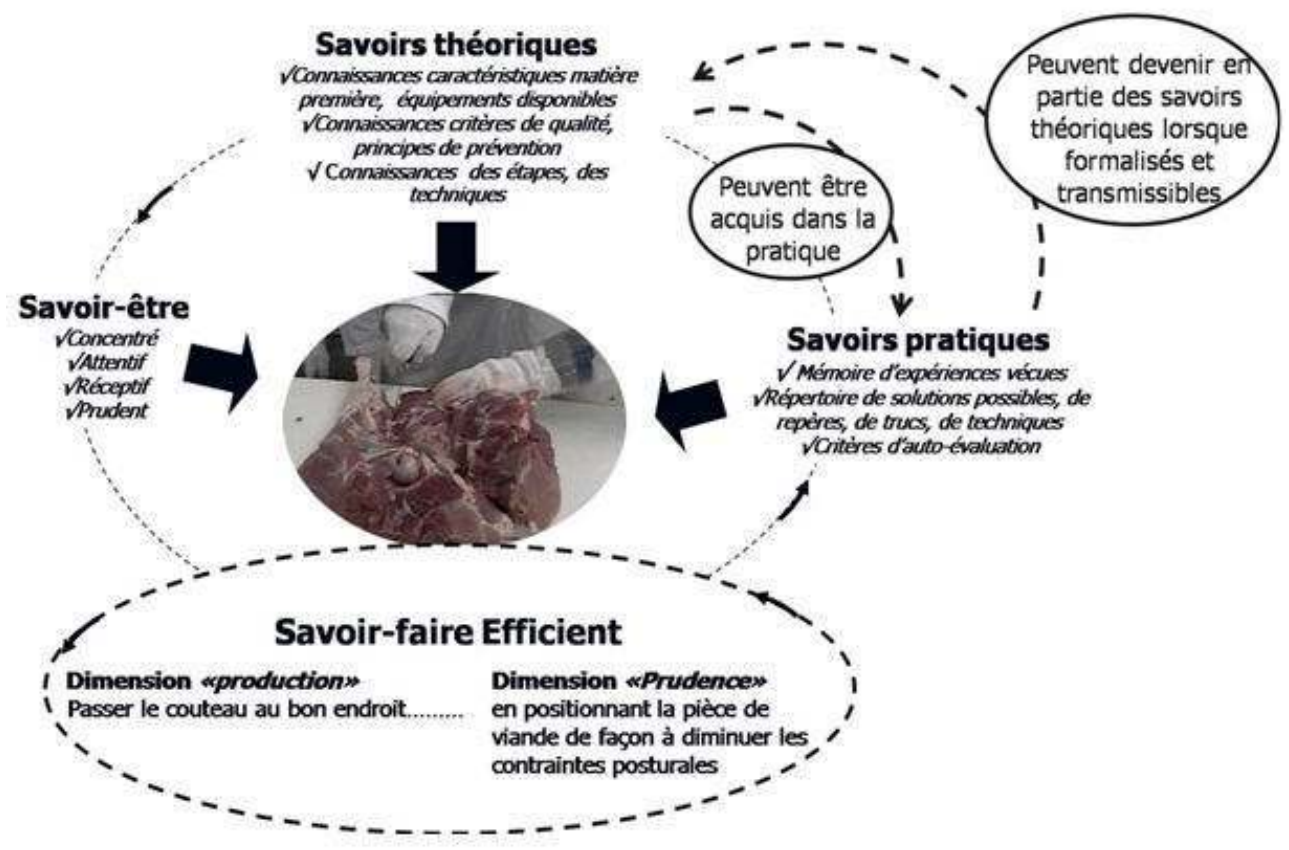

De plus en plus d'études portent sur les questions de formation et de transmission des savoirs (Chatigny et collab., 2006; Fournier, 2003; Cloutier et collab., 2002) et, comme nous l'avons mentionné précédemment lorsqu'il est question de prévention des TMS, l'accent est habituellement mis sur la définition de la dimension prudence des savoirs et des savoir-faire des travailleurs expérimentés dans le but d'en favoriser le développement chez les apprentis. Mais existe-t-il une hiérarchie dans la construction des savoir-faire? Peut-on développer la dimension "prudence» avant d'avoir développé la dimension production? Les données présentées précédemment dans les figures 8 et 9 semblent indiquer que ce n'est pas le cas. Nous avons pu constater que, pour développer le savoirfaire "dégager l'os en donnant un minimum nécessaire de coups de couteau», les travailleurs ont d'abord dû développer deux savoir-faire davantage associés à la production soit "utiliser la partie appropriée de la lame», "passer au bon endroit» et un savoir-faire plus associé à la prudence soit "maintenir la coupe de son couteau ». Ces savoir-faire impliquent eux-mêmes la mobilisation de nombreux savoirs. Il y aurait donc toute une phase de développement cruciale à traverser entre "vouloir être prudent» (savoir-être) et "être capable de l'être» (dimension "prudence»). Cette phase pourrait correspondre à une phase de " pouvoir faire » inspirée de la notion de " pouvoir agir » de Le Boterf (2006, p. 91), déterminée par le contexte et les conditions offertes aux travailleurs pour développer le savoir-faire en question. Il est donc important de considérer l'existence de ces préalables lorsqu'il est question de déterminer comment la formation sera organisée, quel contenu devra être présenté et quelles conditions devront être offertes pour favoriser la construction de ces savoir-faire. La notion de "préalables» suppose nécessairement l'implication du facteur «temps » dans la construction des savoir-faire et de la nécessité d'avoir des attentes réalistes quant à la capacité d'un novice à pouvoir se protéger. 


\subsection{Savoir-faire de production, savoir-faire de prudence ou la convergence des deux?}

Par ailleurs, si nous voulons concevoir des formations visant à prévenir les troubles musculo-squelettiques par la démarche ergonomique, quels types de savoirs et de savoirfaire devrions-nous chercher à définir ? À ce sujet, dans les études en ergonomie visant la prévention des TMS (Denis et collab., 2007; Chassaing, 2006; Chatigny, 2001; Authier, 1996; Gaudart, 1996), l'accent est habituellement mis sur la description des trucs de métier et des stratégies développés par les travailleurs expérimentés pour se protéger (savoir-faire de prudence). Par contre, les résultats obtenus au cours de l'étude montrent que, dans certains cas, des savoir-faire plutôt reconnus comme liés à la production (qualité) peuvent s'avérer aussi très utiles pour diminuer les efforts et la répétition des gestes et que, dans d'autres cas, ce sont des savoir-faire d'abord identifiés pour se protéger qui peuvent être aussi utiles à la production comme par exemple « dégager l'os en donnant le minimum nécessaire de coups de couteau». Dans une perspective de conception de contenus de formation favorisant la prévention des TMS, ne serait-il pas plus approprié d'aborder l'étude des savoir-faire en les situant en fonction de l'impact sur la production et sur la santé et ensuite dégager les savoirs à acquérir pour mettre en œuvre ces savoir-faire. Cette façon intégrée d'aborder la notion de savoir-faire va dans le sens de l'approche ergonomique adoptée dans cette étude qui tient compte du caractère intégrateur de l'activité (Guérin et collab., 2006). Ainsi, ne devrions-nous pas plutôt adopter l'appellation de "savoir-faire efficient " plutôt que de "savoir-faire de prudence » et de "savoir-faire de production ». La " production » et la " prudence » représenteraient les deux dimensions de ce savoir-faire. Un « savoir-faire efficient » serait « la capacité d'un individu à mobiliser dans son activité, un ensemble de savoirs lui permettant de répondre à un objectif visant à la fois la production et la protection de sa santé et celle des autres». La figure 11 présente un exemple de ce que serait l'expression d'un savoir-faire efficient avec ses deux dimensions qui sont celles de la "production » et de la "prudence ».

Figure 11. Exemple d'un savoir-faire efficient dans ses dimensions de «production » et de " prudence »

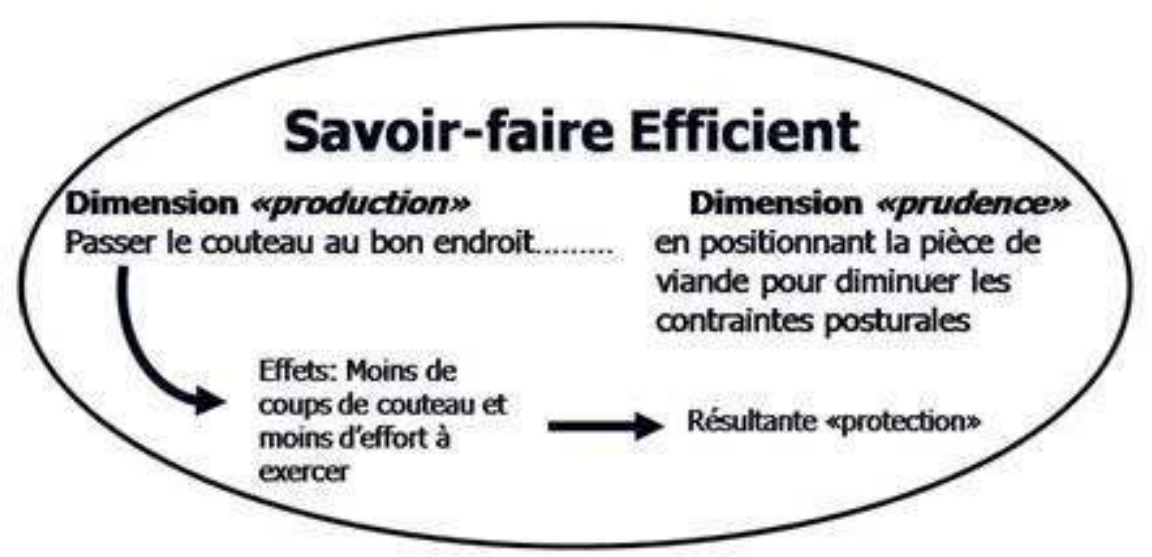




\section{Conclusion}

\subsection{Points forts, limites de l'étude et perspectives de recherche}

La démarche ergonomique développée dans cette étude a été d'un apport important pour décrire les savoir-faire des travailleurs expérimentés, en particulier des deux travailleursformateurs, et favoriser la mise en mots des savoirs. Les étapes d'observation et de verbalisations effectuées auprès d'un groupe de travailleurs expérimentés ont permis d'avoir une compréhension approfondie de l'activité de travail, de faire une description fine des modes opératoires et des gestes effectués par les travailleurs et de favoriser la mise en mots des savoirs pratiques construits au fil du temps pour faciliter le travail. La particularité de cette étude réside surtout dans cette description fine des gestes de travail effectuée à l'intérieur de l'analyse de l'activité qui a mené à une série de questions construites spécifiquement pour la recherche des modalités sensorielles utilisées par les travailleurs dans la réalisation de la tâche. Un point fort de la rencontre collective a été l'utilisation de moyens visuels et d'essais pratiques dans la salle de production qui se sont avérés très instructifs parce qu'ils ont permis aux travailleurs de verbaliser leurs savoirs sur l'action et dans l'action. Il est important de mentionner que la démarche privilégiée dans cette étude ne se voulait pas une démarche déjà existante avec une série d'étapes effectuées dans un ordre rigide, mais plutôt une démarche construite au fil de l'intervention avec l'implication active des différents interlocuteurs de l'entreprise.

L'étude a pu démontrer l'apport de l'analyse ergonomique pour décrire les gestes professionnels tels que définis par Bourgeois et collab. (2006), mais il faut souligner que pour répondre aux questions relatives à des aspects très précis sur les gestes effectués, cette approche présente une certaine limite. En effet, quoique d'une très grande richesse, cette approche se limite à l'analyse qualitative du mouvement effectué, ce qui ne permet pas de fournir des données quantitatives sur la sollicitation musculaire, sur l'angulation précise des segments en cours d'action, la pression exercée par les doigts sur l'outil ainsi que sur la vélocité du mouvement. Ces données auraient pu alimenter les discussions avec les travailleurs dans le but d'aider à la verbalisation des savoirs. Lorsque possible, il serait intéressant d'envisager l'intégration de mesures biomécaniques (Richard, 2002) dans les études visant à élaborer des contenus de formation pour être en mesure de répondre à des questions qui peuvent même être posées par les travailleurs, comme par exemple le niveau de risque présenté par certaines façons de faire telles que la façon de tenir le couteau. L'intérêt de l'interdisciplinarité dans ce genre d'études viendrait du fait que l'analyse qualitative contribuant à décrire la variabilité des gestes professionnels mène à certaines questions auxquelles l'analyse quantitative biomécanique ou physiologique contribuerait en partie à répondre.

De plus, même si nous croyons que la démarche et les méthodes utilisées ont permis la définition de plusieurs savoir-faire et la mise en mots de nombreux savoirs des travailleurs expérimentés, nous ne pouvons prétendre avoir réussi à tout définir. Il n'est probablement pas possible de faire verbaliser tous les savoirs incorporés des travailleurs. Par contre, le recueil de données quantitatives, comme mentionné précédemment, permettrait fort probablement de pousser plus loin cette formalisation des savoirs. De plus, les travailleurs mentionnaient que les rencontres tenues au cours de l'étude les amenaient à réfléchir sur leur pratique. Certains quittaient une rencontre en disant 
vouloir vérifier ce qu'ils faisaient dans certaines circonstances. Nous croyons qu'il serait important de développer une dynamique dans l'entreprise qui favoriserait la poursuite du développement de la formation par l'enrichissement du contenu de formation avec l'intégration d'autres savoirs pratiques découlant de la réflexion continue des travailleurs-formateurs.

\subsection{La portée des résultats de l'étude}

Est-ce qu'une telle démarche privilégiant l'analyse fine des gestes de travail est nécessaire et utile seulement dans des situations où l'on retrouve un travail manuel répétitif? De notre point de vue, peu importe le métier, il y aura toujours un besoin de décrire et de comprendre toutes les composantes des gestes de métier si nous voulons, d'une part, obtenir des contenus de formation représentatifs du travail réel et, d'autre part, favoriser le développement de savoir-faire par la transmission de savoirs de métier. Les travailleurs occupant ce type de postes ont souvent dû apprendre sur le tas et prendre des années, parfois difficiles et souffrantes, pour réussir à développer ces trucs de métier permettant de faciliter le travail. Puisque la souffrance ne devrait aucunement être un passage obligé à l'apprentissage d'une tâche, il s'avère important de faciliter le développement de ces savoir-faire par la transmission des savoirs aux nouveaux travailleurs.

Mentionnons aussi qu'une contribution importante de l'étude réalisée a été de mener à la conception d'un manuel de formation pour le désossage qui a été reçu et validé par les travailleurs-formateurs de l'entreprise (Ouellet, 2006). Une suite à ce projet pourrait être entreprise pour vérifier de quelle façon le contenu de ce manuel pourrait être généralisé dans le secteur de la transformation du porc.

Les auteures remercient la direction de même que les travailleurs, leurs représentants et représentants de l'entreprise qui ont permis la réalisation de ce projet de recherche. Les auteures demeurent seules responsables du contenu de cet article.

\section{BIBLIOGRAPHIE}

Authier, M. (1996). Analyse ergonomique des stratégies de manutentionnaires experts et novices. Thèse de doctorat. Département d'éducation physique. Université de Montréal, Montréal.

Barbier, J.M., Galatanu, O. (2004). Savoirs, capacités, compétences, organisation des champs conceptuels. In Les savoirs d'action : une mise en mots des compétences ?, eds J.M. Barbier, O. Galatanu, p. 31-78. Édition L'Harmattan, Paris.

Berthoz, A. (1997). Le sens du mouvement. Éditions Odile Jacob, Paris, 345 p.

Bouisset, S., (2002). Biomécanique et physiologie du mouvement. Masson, Paris. 304 p.

Bourgeois, F., Kemarchand, C., Hubault, F., Brun, C., Polin, A., Faucheux, J.M. (2006). Troubles musculo-squelettiques et travail - Quand la santé interroge l'organisation. Collection Outils et Méthodes, ANACT. 308 p. 
Bourgeois, F., Hubault, F. (2005). Prévenir les TMS. De la biomécanique à la revalorisation du travail, l'analyse du geste dans toutes ses dimensions. ACTIVITÉS, vol. 2, $\mathrm{n}^{\circ} 1$.

Bril, B., Roux, V. (2002). Le geste technique. Réflexions méthodologiques et anthropologiques. Éditions Éres, Ramonville Saint-Agne. 309 p.

Chassaing, K. (2006). Élaboration, structuration et réalisation des gestuelles de travail : les gestes dans l'assemblage automobile et dans le coffrage des ponts d'autoroute. Thèse de doctorat, spécialité ergonomie. Conservatoire National des Arts et Métiers, Paris, 279 p.

Chatigny, C., Balleux, A., Martin, M., Grenier, J., Ouellet, S., Corbeil, M., Laberge, M., Rochette, D. (2006). Étude exploratoire des dynamiques de formation et d'apprentissage : apprentissage des tâches et prévention des troubles musculo-squelettiques dans trois entreprises du secteur avicole. Études et recherches, IRSST, R-464, Montréal, 80 p., www.irsst.qc.ca/fr/ _publicationirsst_100202.html

Chatigny, C., Vézina, N. (2004). Le développement des compétences : Enjeux de santé et de sécurité au travail. $13^{\mathrm{e}}$ Congrès de psychologie du travail et des organisations, AIPTLF - Bologna, Communication C187, Thème : 4.1 Analyse du travail.

Chatigny, C., (2001a). Polyvalence et développement des compétences : quelques obstacles identifiés lors d'études portant sur les conditions d'apprentissage en entreprise. Comptes rendus $31^{\mathrm{e}}$ Congrès annuel de l'ACE - Ergonomie et sécurité. Hull.

Chatigny, C. (2001b). La construction de ressources opératoires. Construction à la conception des conditions de formation en situation de travail. Thèse de doctorat. Conservatoire National des Arts et Métiers, Paris. 285 p.

Chatigny, C., Vézina, N. (1994). Analyse du travail et apprentissage d'une tâche complexe ; étude de l'affilage du couteau dans un abattoir. Le Travail Humain, 59, p. 229-252.

Chatigny, C. (1993). Étude des conditions d'apprentissage du métier dans un abattoir de porc. Mémoire de maîtrise. Université du Québec à Montréal, Montréal.

Chatigny, C., Vézina, N. (1992). Analyse de l'affilage du couteau dans un abattoir de porc. $25^{\mathrm{e}}$ Congrès de l'Association canadienne d'ergonomie.

Chevallier, D., Chiva, I. (1991). L'introuvable objet de la transmission. In Savoir-faire et pouvoir transmettre. Mission du Patrimoine ethnologique Collection Ethnologique de la France, Cahier 6, ed. D. Chevallier, p. 1-11. Éditions de la Maison des sciences de l'homme, Paris.

Chevallier, D. (1989). Le programme savoir-faire et techniques. Terrain, revue de l'ethnologie de l'Europe, Du congélateur au déménagement. Pratiques de consommation familiale. $\mathrm{n}^{\circ}$ 12, p. 1-6.

Cloutier, E., Lefebvre, S., Ledoux, É., Chatigny, C., St-Jacques, Y. (2002). Enjeux de santé et de sécurité au travail dans la transmission des savoirs professionnels : le cas des usineurs et des cuisiniers. Études et recherches, IRSST, R-316, Montréal, 217 p., www.irsst.qc.ca/fr/ _publicationirsst_859.html

Cru, D. (1995). Règles du métier, langue de métier : dimension symbolique au travail et démarche participative de prévention. Mémoire. École Pratique des Hautes Études. Paris.

Daniellou, F., Garrigou, A. (1995). L'ergonome, l'activité et la parole des travailleurs, In Paroles au travail, J. Boutet, p. 73-92. Éditions L'Harmattan, Paris.

Danis, C., Solar, C. (1998). Apprentissage et développement des adultes. Les Éditions Logiques, Montréal, $319 \mathrm{p}$. 
Deforge, Y. (1991). La transmission et la préservation des savoir-faire et les enseignements techniques. In Chevallier, D. (1991). In Savoir-faire et pouvoir transmettre. Mission du Patrimoine ethnologique Collection Ethnologique de la France, Cahier 6, ed. D. Chevallier, p. 197-216. Éditions de la Maison des sciences de l'homme, Paris.

de Montmollin, M. (1997). Vocabulaire de l'Ergonomie, Éditions Octares, Toulouse, 245 p. de Terssac, G. (1996). La genèse des savoir-faire. In Apprentissages formels et informels dans les organisations, Collection dossiers documentaires, p. 187-199. Édition ANACT, Lyon.

Denis, D., St-Vincent, M., Gonella, M., Couturier, F., Trudeau, R. (2007). Analyse des stratégies de manutention chez des éboueurs au Québec, Pistes de réflexions pour une formation. Études et recherches, IRSST, R-527, Montréal, 80 p., www.irsst.qc.ca/fr/_publicationirsst_100325.html

Doray, P., Dubar, C. (2004). La formation en entreprise au Québec et en France. In Travail et société, Évolution et enjeux, ed. D.-G. Tremblay, p. 301-319. Presses de l'Université du Québec, Sainte-Foy.

Dubé, A., Mercure, D. (1997). Les entreprises et l'emploi. Les nouvelles formes de qualification du travail. Les Publications du Québec, Québec. 189 p.

Durand, M.J., Vézina, N., Baril, R., Loisel, P., Richard, M.-C., Ngomo, S. (2008). Étude exploratoire sur la marge de manœuvre de travailleurs pendant et après un programme de retour progressif au travail : définition et relation(s) avec le retour en emploi. Collection Études et Recherches, IRSST, Projet 099-477, Montréal, 65 p.

Everaere, C. (1999). Autonomie et collectif de travail. Collection Points de Repère, ANACT, p. 7-20. Fournier, P.-S. (2003). L'aménagement de situations d'action sur le cours de vie professionnelle du camionneur : un apport à la démarche de conception d'une formation initiale en lien avec l'activité de travail. Thèse de doctorat. Université Laval. Québec. 345 p.

Garrigou, A., Peeters, S., Jackson, M., Sagory, P., Carballera, G. (2004). In Ergonomie, ed. P. Falzon, p. 497-514. Presses Universitaires de France, Paris.

Gaudart, C. (1996). Transformations de l'activité avec l'âge dans des tâches de montage automobile sur chaîne. Thèse de doctorat d'ergonomie. École Pratique des Hautes Études, Paris, $215 \mathrm{p}$.

Guérin, F., Laville, A., Daniellou, F., Duraffourg, J., Kerguelen, A. (2006). Comprendre le travail pour le transformer. La pratique de l'ergonomie. (1 ${ }^{\text {re }}$ édition. $1991,2^{\mathrm{e}}$ édition, 1997) $3^{\mathrm{e}}$ édition. ANACT. 287 p.

Kerguelen, A. (2003). Actogram Kronos pour Windows, Éditions Octares, Toulouse.

Lachance, R. (2000). L'importance des mécanismes de régulation temporelle dans le travail des opératrices de métiers à fibres libérées relativement à l'automatisation des machines et au travail de nuit. PISTES, vol. 2, $\mathrm{n}^{\circ}$ 2, www.pistes.uqam.ca/v2n2/articles/v2n2a1.htm

Laflamme, R. (2002). La formation en entreprise : Nécessité ou contrainte ? Les Presses de l'Université Laval, Saint-Nicolas, 199 p.

Latash, M.L., Turvey, M.T. (1996). Dexterity and its development. With On Dexterity and its development by Nicholai A. Bernstein. LEA Lawrence Erlbaum Associates Inc., 460 p.

Le Boterf, G. (2006). Construire les compétences individuelles et collectives. Agir et réussir avec compétence. $4^{\mathrm{e}}$ édition mise à jour et complétée, Éditions d'Organisation, Paris, $271 \mathrm{p}$.

Leplat, J. (2005). Les automatismes dans l'activité : pour une réhabilitation et un bon usage. Activités, vol 2, n 2, http://www.activites.org/v2n2/leplat.pdf 
Minet, F. (2005). Compétence : de la définition à l'utilisation. In Élaborer des référentiels de compétences, eds C. Jouvenot, M. Parlier, p. 332-362. Éditions ANACT, Lyon.

Murray, G., Bélanger, J., Giles, A., Lapointe, P.-A. (2004). L'organisation de la production et du travail : vers un nouveau modèle ?, p. 29-35. Les Presses de l'Université Laval, Québec.

Organisation mondiale de la santé. Constitution de l'Organisation Mondiale de la Santé (1948), 20 p., /www.who.int/governance/eb/who_constitution_fr.pdf

Ouellet, S. (2006). Manuel de formation au dégraissage et au désossage de fesses de porc, Manuel préparé suite à une intervention réalisée dans le cadre d'un projet de doctorat. Université du Québec à Montréal, Montréal, $125 \mathrm{p}$.

Ouellet, S., Vézina N., Chartrand, J., Perrier P.-P., Malo J.-L. (2003). L'implantation de la rotation de postes : un exemple de démarche préalable. PISTES, vol. $5, n^{\circ} 2$, www.pistes.uqam.ca/v5n2/ articles/v5n2a14.htm

Ouellet, S., Vézina, N. (2001). Analyse des déterminants de la qualité de coupe du whizard : vers la concertation des actions et l'élaboration d'une formation. Comptes rendus du $36^{\mathrm{e}}$ Congrès de la Société d'ergonomie de langue française et du $32^{\mathrm{e}}$ Congrès de l'Association canadienne d'ergonomie - Les transformations du travail : enjeux pour l'ergonomie. Montréal, Québec, Canada. p. 1-6.

Ouellet, S. (2000). Étude ergonomique au poste de dégraisseur dans une usine de transformation du porc. Rapport, Département des sciences biologiques, Université du Québec à Montréal. Disponible au Centre de documentation de la Commission de la santé et de la sécurité du travail (CSST). Cote MO-022109. Montréal, 218 p.

Pastré, P. (2004). Recherches en didactique professionnelle. In Recherches en didactique professionnelle, eds R. Samurçay, P. Pastré, p. 4. Éditions Octarès, Toulouse.

Pelegrin, J. (1991). Les savoir-faire : une très longue histoire, Terrain, Revue d'ethnologie de l'Europe, $\mathrm{n}^{\circ} 16$, p. 1-13.

Rasse, P. (1991). La cité aromatique. Culture, techniques et savoir-faire dans les industries de la parfumerie grassoise. Terrain, revue d'ethnologie de l'Europe, $n^{\circ} 16$, p. 1-27.

Reber, A. S. (1989). Implicit learning and tacit knowledge. Journal of Experimental Psychology, General, nº 118, p. 219-235.

Richard, P. (2002). Analyse ergonomique et mesures biomécaniques dans un abattoir de porcs. PISTES, vol 4, $\mathrm{n}^{\circ}$ 1, www.pistes.uqam.ca/v4n1/articles/v4n1a3.htm

Rouilleault, H., Rochefort, T. (2005). Changer le travail... oui mais ensemble. ANACT, Lyon, p. 20-51.

Schmidt, R.A., Lee T.D., (2005). Motor control and learning. A behavioral emphasis. $4^{\mathrm{e}}$ edition, $\left(1^{\mathrm{re}}\right.$ édition 1982; $2^{\mathrm{e}}$ 1988; $3^{\mathrm{e}}$ édition, 1999), Human Kinetics, Champaign, $536 \mathrm{p}$.

Sperandio, J.-C. (1996). L'ergonomie face aux changements technologiques et organisationnels du travail humain. Éditions Octares, p. 11 à 25.

Stroobants, M. (1993). La mobilisation des compétences. In Savoir-faire et compétences au travail. Une sociologie de la fabrication des aptitudes, ed. M. Stroobants, p. 47-63. Éditions de l'Université de Bruxelles, Belgique.

Teiger, C. (1996). L'approche ergonomique : de travail humain à l'activité des hommes et des femmes au travail. In Apprentissages formels et informels dans les organisations, p. 109-125. Éditions ANACT, Lyon. 
Teiger, C. (1993). Représentation du travail et travail de représentation. In Représentations pour l'action, eds A. Weill-Fassina, P. Rabardel, D. Dubois, p. 311-340. Éditions OCTARES, Toulouse.

Teiger, C., Laville, A. (1989). Expression des travailleurs sur leurs conditions de travail. Rapport $\mathrm{n}^{\circ} 100$. Laboratoire d'ergonomie. CNAM, Paris.

Theureau, J. (1991). Cours d'action et savoir-faire. In Savoir-faire et pouvoir transmettre. Mission du Patrimoine ethnologique Collection Ethnologique de la France, Cahier 6, ed. D. Chevallier, p. 43-60. Éditions de la Maison des sciences de l'homme, Paris.

Vézina, N., Ouellet, S. (2002). Élaboration d'une méthode standardisée d'évaluation de la qualité de coupe des couteaux. Rapport déposé à la Commission de la santé et de la sécurité du travail (CSST). Montréal.

Vézina, N., Ouellet, S., Richard, P., Léveillée, M.-S. (2002). Procédure d'implantation de la formation à l'affilage des couteaux. Document déposé au Fonds québécois d'adaptation des entreprises agroalimentaires (FQAEA).

Vézina, N., (2001). La pratique de l'ergonomie face aux TMS : ouverture à l'interdisciplinarité. Comptes rendus du congrès SELF-ACE 2001, Les transformations du travail, enjeux pour l'ergonomie. http://www.ergonomie-self.org/documents/36eme-Montreal-2001/PDF-FR/v1-05avezina.pdf

Vézina, N., Prévost, J., Lajoie, A., Beauchamp, Y. (1999). Élaboration d'une formation à l'affilage des couteaux : le travail d'un collectif, travailleurs et ergonomes. PISTES, vol. 1, $\mathrm{n}^{\circ} 1$, www.pistes.uqam.ca/v1n1/articles/v1n1a3.htm

Vidal-Gomel, C. (2002). Systèmes d'instruments des opérateurs. Un point de vue pour analyser le rapport aux règles de sécurité. PISTES, vol. $4, \mathrm{n}^{\circ}$ 2, www.pistes.uqam.ca/v4n2/articles/ v4n2a2.htm

\section{RÉSUMÉS}

Le travail répétitif est souvent vu comme un travail qui peut s'apprendre par la simple observation d'un collègue. Cette étude a eu pour objectif de décrire les savoir-faire et en particulier ceux qui permettent de se protéger, qui sont développés par six travailleurs-experts d'un département de découpe de la viande. Des entretiens individuels et collectifs, des observations sur place et sur vidéos et des rencontres d'autoconfrontation ont été réalisés. Une typologie des savoirs à la base des savoir-faire a été élaborée et l'existence d'une hiérarchie dans la construction des savoir-faire a été dévoilée, notamment pour des savoir-faire appelés « savoirfaire efficients ». Cette hiérarchie fait ressortir toute la complexité du travail qui devrait être considérée dans l'organisation de la formation et la présentation du contenu. La démarche ergonomique développée dans cette étude ainsi qu'un cadre théorique issu de la pratique sur le terrain ont permis de mettre au jour la richesse des savoirs mobilisés dans le travail manuel.

Repetitive work is often seen as work that can be learned by simple observation of a colleague. This study aimed to describe the know-how and especially the know-how that can be used for one's protection, developed by six expert workers in a meat-cutting department. Individual and group interviews, on-site and video observations, and individual auto-confrontation (confronting participants with their own activity) were conducted. A typology of the know-how's knowledge base was developed and the existence of a hierarchy in the development of expertise was brought to light, including know-how called «efficient know-how ». This hierarchy highlights all the complexity of the work that should be considered in organizing training and presenting content. 
The ergonomic approach developed in this study and a theoretical framework resulting from practice have helped to uncover the wealth of knowledge that is mobilized in manual labor.

El trabajo repetitivo está visto a menudo como un trabajo que puede aprenderse a traves de la simple observacion de un colega. Este trabajo intenta describir los saber-hacer y en particular aquellos que permiten protegerse, desarrollados por seis trabajadores expertos de un departamento de corte de carne. Se han realizado entrevistas individuales y colectivas, observaciones in situ y en base a videos, asi como encuentros de auto-confrontación. Una tipología de saberes fundacionales de los saber-hacer a sido abordada y la existencia de una jerarquía en la construcción de los saber-hacer ha sido develada, sobre todo para los saber-hacer llamados «saber-hacer eficientes ». Esta jerarquía hace resaltar toda la complejidad del trabajo que debería ser considerado en la organización de la formación y de la presentación del contenido. El enfoque ergonómico desarrollado en este estudio, asi como también un marco teórico inspirado en la práctica de campo, permitieron poner al día la riqueza de los saberes mobilizados en el trabajo manual.

\section{INDEX}

Mots-clés : ergonomie, troubles musculo-squelettiques, formation, savoir, savoir-faire

Palabras claves : ergonomía, lesiones músculo-esqueléticas, capacitación, saber, saber-hacer

Keywords : ergonomic, musculoskeletal disorders, training, knowledge, know how

\section{AUTEURS}

\section{SYLVIE OUELLET}

CINBIOSE, Université du Québec à Montréal, Montréal (Québec)ouellet.sylvie@uqam.ca

\section{NICOLE VÉZINA}

CINBIOSE, Université du Québec à Montréal, Montréal (Québec)vezina.nicole@uqam.ca 